Osvaldo Gervasi • Beniamino Murgante • Sanjay Misra • Chiara Garau • Ivan Blečić • David Taniar · Bernady O. Apduhan • Ana Maria A. C. Rocha - Eufemia Tarantino • Carmelo Maria Torre (Eds.)

\title{
Computational Science and Its Applications - ICCSA 2021
}

21st International Conference Cagliari, Italy, September 13-16, 2021 Proceedings, Part VI

\section{Part VI}

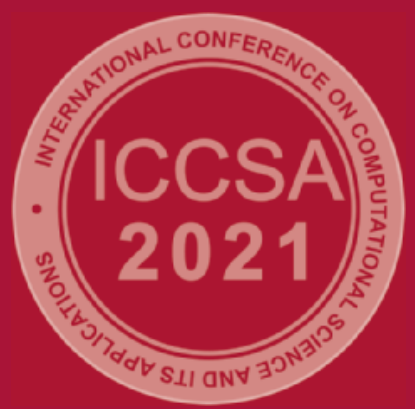

Springer 


\section{Lecture Notes in Computer Science}

Founding Editors

Gerhard Goos

Karlsruhe Institute of Technology, Karlsruhe, Germany

Juris Hartmanis

Cornell University, Ithaca, NY, USA

Editorial Board Members

Elisa Bertino

Purdue University, West Lafayette, IN, USA

Wen Gao

Peking University, Beijing, China

Bernhard Steffen (1)

TU Dortmund University, Dortmund, Germany

Gerhard Woeginger (1)

RWTH Aachen, Aachen, Germany

Moti Yung

Columbia University, New York, NY, USA 
More information about this subseries at http://www.springer.com/series/7407 
Osvaldo Gervasi · Beniamino Murgante ·

Sanjay Misra - Chiara Garau .

Ivan Blečić - David Taniar .

Bernady O. Apduhan - Ana Maria A. C. Rocha .

Eufemia Tarantino - Carmelo Maria Torre (Eds.)

\section{Computational Science and Its Applications - ICCSA 2021}

21st International Conference

Cagliari, Italy, September 13-16, 2021

Proceedings, Part VI

黛 Springer 


\section{Editors}

Osvaldo Gervasi (D)

University of Perugia

Perugia, Italy

Sanjay Misra 1 iD

Covenant University

Ota, Nigeria

Ivan Blečić (iD)

University of Cagliari

Cagliari, Italy

Bernady O. Apduhan

Kyushu Sangyo University

Fukuoka, Japan

Eufemia Tarantino (D)

Polytechnic University of Bari

Bari, Italy
Beniamino Murgante (D)

University of Basilicata

Potenza, Potenza, Italy

Chiara Garau (D)

University of Cagliari

Cagliari, Italy

David Taniar (iD

Monash University

Clayton, VIC, Australia

Ana Maria A. C. Rocha (D)

University of Minho

Braga, Portugal

Carmelo Maria Torre

Polytechnic University of Bari

Bari, Italy

ISSN 0302-9743

ISSN 1611-3349 (electronic)

Lecture Notes in Computer Science

ISBN 978-3-030-86978-6

ISBN 978-3-030-86979-3 (eBook)

https://doi.org/10.1007/978-3-030-86979-3

LNCS Sublibrary: SL1 - Theoretical Computer Science and General Issues

(C) Springer Nature Switzerland AG 2021

This work is subject to copyright. All rights are reserved by the Publisher, whether the whole or part of the material is concerned, specifically the rights of translation, reprinting, reuse of illustrations, recitation, broadcasting, reproduction on microfilms or in any other physical way, and transmission or information storage and retrieval, electronic adaptation, computer software, or by similar or dissimilar methodology now known or hereafter developed.

The use of general descriptive names, registered names, trademarks, service marks, etc. in this publication does not imply, even in the absence of a specific statement, that such names are exempt from the relevant protective laws and regulations and therefore free for general use.

The publisher, the authors and the editors are safe to assume that the advice and information in this book are believed to be true and accurate at the date of publication. Neither the publisher nor the authors or the editors give a warranty, expressed or implied, with respect to the material contained herein or for any errors or omissions that may have been made. The publisher remains neutral with regard to jurisdictional claims in published maps and institutional affiliations.

This Springer imprint is published by the registered company Springer Nature Switzerland AG The registered company address is: Gewerbestrasse 11, 6330 Cham, Switzerland 


\section{Preface}

These 10 volumes (LNCS volumes 12949-12958) consist of the peer-reviewed papers from the 21st International Conference on Computational Science and Its Applications (ICCSA 2021) which took place during September 13-16, 2021. By virtue of the vaccination campaign conducted in various countries around the world, we decided to try a hybrid conference, with some of the delegates attending in person at the University of Cagliari and others attending in virtual mode, reproducing the infrastructure established last year.

This year's edition was a successful continuation of the ICCSA conference series, which was also held as a virtual event in 2020, and previously held in Saint Petersburg, Russia (2019), Melbourne, Australia (2018), Trieste, Italy (2017), Beijing. China (2016), Banff, Canada (2015), Guimaraes, Portugal (2014), Ho Chi Minh City, Vietnam (2013), Salvador, Brazil (2012), Santander, Spain (2011), Fukuoka, Japan (2010), Suwon, South Korea (2009), Perugia, Italy (2008), Kuala Lumpur, Malaysia (2007), Glasgow, UK (2006), Singapore (2005), Assisi, Italy (2004), Montreal, Canada (2003), and (as ICCS) Amsterdam, The Netherlands (2002) and San Francisco, USA (2001).

Computational science is the main pillar of most of the present research on understanding and solving complex problems. It plays a unique role in exploiting innovative ICT technologies and in the development of industrial and commercial applications. The ICCSA conference series provides a venue for researchers and industry practitioners to discuss new ideas, to share complex problems and their solutions, and to shape new trends in computational science.

Apart from the six main conference tracks, ICCSA 2021 also included 52 workshops in various areas of computational sciences, ranging from computational science technologies to specific areas of computational sciences, such as software engineering, security, machine learning and artificial intelligence, blockchain technologies, and applications in many fields. In total, we accepted 494 papers, giving an acceptance rate of $30 \%$, of which 18 papers were short papers and 6 were published open access. We would like to express our appreciation for the workshop chairs and co-chairs for their hard work and dedication.

The success of the ICCSA conference series in general, and of ICCSA 2021 in particular, vitally depends on the support of many people: authors, presenters, participants, keynote speakers, workshop chairs, session chairs, organizing committee members, student volunteers, Program Committee members, advisory committee members, international liaison chairs, reviewers, and others in various roles. We take this opportunity to wholehartedly thank them all.

We also wish to thank Springer for publishing the proceedings, for sponsoring some of the best paper awards, and for their kind assistance and cooperation during the editing process. 
We cordially invite you to visit the ICCSA website https://iccsa.org where you can find all the relevant information about this interesting and exciting event.

September 2021

Osvaldo Gervasi

Beniamino Murgante

Sanjay Misra 


\section{Welcome Message from the Organizers}

COVID-19 has continued to alter our plans for organizing the ICCSA 2021 conference, so although vaccination plans are progressing worldwide, the spread of virus variants still forces us into a period of profound uncertainty. Only a very limited number of participants were able to enjoy the beauty of Sardinia and Cagliari in particular, rediscovering the immense pleasure of meeting again, albeit safely spaced out. The social events, in which we rediscovered the ancient values that abound on this wonderful island and in this city, gave us even more strength and hope for the future. For the management of the virtual part of the conference, we consolidated the methods, organization, and infrastructure of ICCSA 2020.

The technological infrastructure was based on open source software, with the addition of the streaming channels on YouTube. In particular, we used Jitsi (jitsi.org) for videoconferencing, Riot (riot.im) together with Matrix (matrix.org) for chat and ansynchronous communication, and Jibri (github.com/jitsi/jibri) for streaming live sessions to YouTube.

Seven Jitsi servers were set up, one for each parallel session. The participants of the sessions were helped and assisted by eight student volunteers (from the universities of Cagliari, Florence, Perugia, and Bari), who provided technical support and ensured smooth running of the conference proceedings.

The implementation of the software infrastructure and the technical coordination of the volunteers were carried out by Damiano Perri and Marco Simonetti.

Our warmest thanks go to all the student volunteers, to the technical coordinators, and to the development communities of Jitsi, Jibri, Riot, and Matrix, who made their terrific platforms available as open source software.

A big thank you goes to all of the 450 speakers, many of whom showed an enormous collaborative spirit, sometimes participating and presenting at almost prohibitive times of the day, given that the participants of this year's conference came from 58 countries scattered over many time zones of the globe.

Finally, we would like to thank Google for letting us stream all the live events via YouTube. In addition to lightening the load of our Jitsi servers, this allowed us to record the event and to be able to review the most exciting moments of the conference.

Ivan Blečić

Chiara Garau 


\section{Organization}

ICCSA 2021 was organized by the University of Cagliari (Italy), the University of Perugia (Italy), the University of Basilicata (Italy), Monash University (Australia), Kyushu Sangyo University (Japan), and the University of Minho (Portugal).

\section{Honorary General Chairs}

Norio Shiratori

Kenneth C. J. Tan

Corrado Zoppi

\section{General Chairs}

Osvaldo Gervasi

Ivan Blečić

David Taniar
Chuo University, Japan

Sardina Systems, UK

University of Cagliari, Italy

\section{Program Committee Chairs}

Beniamino Murgante

Bernady O. Apduhan

Chiara Garau

Ana Maria A. C. Rocha
University of Perugia, Italy

University of Cagliari, Italy

Monash University, Australia

\section{International Advisory Committee}

Jemal Abawajy

Dharma P. Agarwal

Rajkumar Buyya

Claudia Bauzer Medeiros

Manfred M. Fisher

Marina L. Gavrilova

Yee Leung
University of Basilicata, Italy

Kyushu Sangyo University, Japan

University of Cagliari, Italy

University of Minho, Portugal

\section{International Liaison Chairs}

Giuseppe Borruso

Elise De Donker

Maria Irene Falcão

Robert C. H. Hsu

Tai-Hoon Kim
Deakin University, Australia

University of Cincinnati, USA

University of Melbourne, Australia

University of Campinas, Brazil

Vienna University of Economics and Business, Austria University of Calgary, Canada

Chinese University of Hong Kong, China
University of Trieste, Italy

Western Michigan University, USA

University of Minho, Portugal

Chung Hua University, Taiwan

Beijing Jaotong University, China 
Vladimir Korkhov

Sanjay Misra

Takashi Naka

Rafael D. C. Santos

Maribel Yasmina Santos

Elena Stankova
St. Petersburg University, Russia

Covenant University, Nigeria

Kyushu Sangyo University, Japan

National Institute for Space Research, Brazil

University of Minho, Portugal

St. Petersburg University, Russia

\section{Workshop and Session Chairs}

Beniamino Murgante

Sanjay Misra

Jorge Gustavo Rocha
University of Basilicata, Italy

Covenant University, Nigeria

University of Minho, Portugal

\section{Awards Chair}

Wenny Rahayu

La Trobe University, Australia

\section{Publicity Committee Chairs}

Elmer Dadios

Nataliia Kulabukhova

Daisuke Takahashi

Shangwang Wang

\section{Technology Chairs}

Damiano Perri

Marco Simonetti
De La Salle University, Philippines

St. Petersburg University, Russia

Tsukuba University, Japan

Beijing University of Posts and Telecommunications, China

\section{Local Arrangement Chairs}

Ivan Blečić

Chiara Garau

Alfonso Annunziata

Ginevra Balletto

Giuseppe Borruso

Alessandro Buccini

Michele Campagna

Mauro Coni

Anna Maria Colavitti

Giulia Desogus

Caterina Fenu

Sabrina Lai

Francesca Maltinti

Pasquale Mistretta
University of Florence, Italy

University of Florence, Italy
University of Cagliari, Italy

University of Cagliari, Italy

University of Cagliari, Italy

University of Cagliari, Italy

University of Trieste, Italy

University of Cagliari, Italy

University of Cagliari, Italy

University of Cagliari, Italy

University of Cagliari, Italy

University of Cagliari, Italy

University of Cagliari, Italy

University of Cagliari, Italy

University of Cagliari, Italy

University of Cagliari, Italy 
Augusto Montisci

Francesco Pinna

Davide Spano

Giuseppe A. Trunfio

Corrado Zoppi

\section{Program Committee}

\author{
Vera Afreixo \\ Filipe Alvelos \\ Hartmut Asche \\ Ginevra Balletto \\ Michela Bertolotto \\ Sandro Bimonte \\ Rod Blais \\ Ivan Blečić \\ Giuseppe Borruso \\ Ana Cristina Braga \\ Massimo Cafaro \\ Yves Caniou \\ José A. Cardoso e Cunha \\ Rui Cardoso \\ Leocadio G. Casado \\ Carlo Cattani \\ Mete Celik \\ Maria Cerreta \\ Hyunseung Choo \\ Chien-Sing Lee \\ Min Young Chung \\ Florbela Maria da Cruz \\ Domingues Correia \\ Gilberto Corso Pereira \\ Fernanda Costa \\ Alessandro Costantini \\ Carla Dal Sasso Freitas \\ Pradesh Debba
}

Hendrik Decker

Robertas Damaševičius

Frank Devai

Rodolphe Devillers

Joana Matos Dias

Paolino Di Felice

Prabu Dorairaj

Noelia Faginas Lago

M. Irene Falcao
University of Cagliari, Italy

University of Cagliari, Italy

University of Cagliari, Italy

University of Sassari, Italy

University of Cagliari, Italy
University of Aveiro, Portugal

University of Minho, Portugal

University of Potsdam, Germany

University of Cagliari, Italy

University College Dublin, Ireland

INRAE-TSCF, France

University of Calgary, Canada

University of Sassari, Italy

University of Trieste, Italy

University of Minho, Portugal

University of Salento, Italy

University of Lyon, France

Universidade Nova de Lisboa, Portugal

University of Beira Interior, Portugal

University of Almeria, Spain

University of Salerno, Italy

Erciyes University, Turkey

University of Naples "Federico II", Italy

Sungkyunkwan University, South Korea

Sunway University, Malaysia

Sungkyunkwan University, South Korea

Polytechnic Institute of Viana do Castelo, Portugal

Federal University of Bahia, Brazil

University of Minho, Portugal

INFN, Italy

Universidade Federal do Rio Grande do Sul, Brazil

The Council for Scientific and Industrial Research (CSIR), South Africa

Instituto Tecnolčgico de Informática, Spain

Kausan University of Technology, Lithuania

London South Bank University, UK

Memorial University of Newfoundland, Canada

University of Coimbra, Portugal

University of L'Aquila, Italy

NetApp, India/USA

University of Perugia, Italy

University of Minho, Portugal 
Cherry Liu Fang

Florbela P. Fernandes

Jose-Jesus Fernandez

Paula Odete Fernandes

Adelaide de Fátima Baptista

Valente Freitas

Manuel Carlos Figueiredo

Maria Celia Furtado Rocha

Chiara Garau

Paulino Jose Garcia Nieto

Jerome Gensel

Maria Giaoutzi

Arminda Manuela Andrade

Pereira Gonçalves

Andrzej M. Goscinski

Eduardo Guerra

Sevin Gümgüm

Alex Hagen-Zanker

Shanmugasundaram

Hariharan

Eligius M. T. Hendrix

Hisamoto Hiyoshi

Mustafa Inceoglu

Peter Jimack

Qun Jin

Yeliz Karaca

Farid Karimipour

Baris Kazar

Maulana Adhinugraha Kiki

DongSeong Kim

Taihoon Kim

Ivana Kolingerova

Nataliia Kulabukhova

Vladimir Korkhov

Rosa Lasaponara

Maurizio Lazzari

Cheng Siong Lee

Sangyoun Lee

Jongchan Lee

Chendong $\mathrm{Li}$

Gang Li

Fang Liu

Xin Liu

Andrea Lombardi

Savino Longo
Ames Laboratory, USA

Polytechnic Institute of Bragança, Portugal

National Centre for Biotechnology, Spain

Polytechnic Institute of Bragança, Portugal

University of Aveiro, Portugal

University of Minho, Portugal

Universidade Federal da Bahia, Brazil

University of Cagliari, Italy

University of Oviedo, Spain

LSR-IMAG, France

National Technical University of Athens, Greece

University of Minho, Portugal

Deakin University, Australia

Free University of Bozen-Bolzano, Italy

Izmir University of Economics, Turkey

University of Cambridge, UK

B.S. Abdur Rahman University, India

University of Malaga, Spain/Wageningen University, The Netherlands

Gunma University, Japan

EGE University, Turkey

University of Leeds, UK

Waseda University, Japan

University of Massachusetts Medical School, USA

Vienna University of Technology, Austria

Oracle Corp., USA

Telkom University, Indonesia

University of Canterbury, New Zealand

Hannam University, South Korea

University of West Bohemia, Czech Republic

St. Petersburg University, Russia

St. Petersburg University, Russia

National Research Council, Italy

National Research Council, Italy

Monash University, Australia

Yonsei University, South Korea

Kunsan National University, South Korea

University of Connecticut, USA

Deakin University, Australia

Ames Laboratory, USA

University of Calgary, Canada

University of Perugia, Italy

University of Bari, Italy 
Tinghuai Ma

Ernesto Marcheggiani

Antonino Marvuglia

Nicola Masini

Ilaria Matteucci

Eric Medvet

Nirvana Meratnia

Giuseppe Modica

Josè Luis Montaña

Maria Filipa Mourão

Louiza de Macedo Mourelle

Nadia Nedjah

Laszlo Neumann

Kok-Leong Ong

Belen Palop

Marcin Paprzycki

Eric Pardede

Kwangjin Park

Ana Isabel Pereira

Massimiliano Petri

Telmo Pinto

Maurizio Pollino

Alenka Poplin

Vidyasagar Potdar

David C. Prosperi

Wenny Rahayu

Jerzy Respondek

Humberto Rocha

Jon Rokne

Octavio Roncero

Maytham Safar

Francesco Santini

Chiara Saracino

Haiduke Sarafian

Marco Paulo Seabra dos Reis

Jie Shen

Qi Shi

Dale Shires

Inês Soares

Elena Stankova

Takuo Suganuma

Eufemia Tarantino

Sergio Tasso
Nanjing University of Information Science and Technology, China

Katholieke Universiteit Leuven, Belgium

Research Centre Henri Tudor, Luxembourg

National Research Council, Italy

National Research Council, Italy

University of Trieste, Italy

University of Twente, The Netherlands

University of Reggio Calabria, Italy

University of Cantabria, Spain

Instituto Politécnico de Viana do Castelo, Portugal

State University of Rio de Janeiro, Brazil

State University of Rio de Janeiro, Brazil

University of Girona, Spain

Deakin University, Australia

Universidad de Valladolid, Spain

Polish Academy of Sciences, Poland

La Trobe University, Australia

Wonkwang University, South Korea

Polytechnic Institute of Bragança, Portugal

University of Pisa, Italy

University of Coimbra, Portugal

Italian National Agency for New Technologies, Energy and Sustainable Economic Development, Italy

University of Hamburg, Germany

Curtin University of Technology, Australia

Florida Atlantic University, USA

La Trobe University, Australia

Silesian University of Technology Poland

INESC-Coimbra, Portugal

University of Calgary, Canada

CSIC, Spain

Kuwait University, Kuwait

University of Perugia, Italy

A.O. Ospedale Niguarda Ca' Granda, Italy

Pennsylvania State University, USA

University of Coimbra, Portugal

University of Michigan, USA

Liverpool John Moores University, UK

U.S. Army Research Laboratory, USA

University of Coimbra, Portugal

St. Petersburg University, Russia

Tohoku University, Japan

Polytechnic University of Bari, Italy

University of Perugia, Italy 
Ana Paula Teixeira

Senhorinha Teixeira

M. Filomena Teodoro

Parimala Thulasiraman

Carmelo Torre

Javier Martinez Torres

Giuseppe A. Trunfio

Pablo Vanegas

Marco Vizzari

Varun Vohra

Koichi Wada

Krzysztof Walkowiak

Zequn Wang

Robert Weibel

Frank Westad

Roland Wismüller

Mudasser Wyne

Chung-Huang Yang

Xin-She Yang

Salim Zabir

Haifeng Zhao

Fabiana Zollo

Albert Y. Zomaya
University of Trás-os-Montes and Alto Douro, Portugal

University of Minho, Portugal

Portuguese Naval Academy/University of Lisbon, Portugal

University of Manitoba, Canada

Polytechnic University of Bari, Italy

Centro Universitario de la Defensa Zaragoza, Spain

University of Sassari, Italy

University of Cuenca, Equador

University of Perugia, Italy

Merck Inc., USA

University of Tsukuba, Japan

Wroclaw University of Technology, Poland

Intelligent Automation Inc, USA

University of Zurich, Switzerland

Norwegian University of Science and Technology, Norway

Universität Siegen, Germany

National University, USA

National Kaohsiung Normal University, Taiwan

National Physical Laboratory, UK

National Institute of Technology, Tsuruoka, Japan

University of California, Davis, USA

University of Venice "Cà Foscari”, Italy

University of Sydney, Australia

\section{Workshop Organizers}

Advanced Transport Tools and Methods (A2TM 2021)

Massimiliano Petri

University of Pisa, Italy

Antonio Pratelli

University of Pisa, Italy

Advances in Artificial Intelligence Learning Technologies: Blended Learning, STEM, Computational Thinking and Coding (AAILT 2021)
Alfredo Milani
University of Perugia, Italy
Giulio Biondi
University of Florence, Italy
Sergio Tasso
University of Perugia, Italy

Workshop on Advancements in Applied Machine Learning and Data Analytics (AAMDA 2021)
Alessandro Costantini
INFN, Italy
Davide Salomoni
INFN, Italy
Doina Cristina Duma
INFN, Italy
Daniele Cesini
INFN, Italy 
Automatic Landform Classification: Spatial Methods and Applications (ALCSMA 2021)
Maria Danese
ISPC, National Research Council, Italy
Dario Gioia
ISPC, National Research Council, Italy

Application of Numerical Analysis to Imaging Science (ANAIS 2021)

Caterina Fenu

Alessandro Buccini
University of Cagliari, Italy

University of Cagliari, Italy

Advances in Information Systems and Technologies for Emergency Management, Risk Assessment and Mitigation Based on the Resilience Concepts (ASTER 2021)
Maurizio Pollino
ENEA, Italy
Marco Vona
University of Basilicata, Italy
Amedeo Flora
University of Basilicata, Italy
Chiara Iacovino
University of Basilicata, Italy
Beniamino Murgante
University of Basilicata, Italy

Advances in Web Based Learning (AWBL 2021)

Birol Ciloglugil Ege University, Turkey

Mustafa Murat Inceoglu Ege University, Turkey

Blockchain and Distributed Ledgers: Technologies and Applications (BDLTA 2021)
Vladimir Korkhov
St. Petersburg University, Russia
Elena Stankova
St. Petersburg University, Russia
Nataliia Kulabukhova
St. Petersburg University, Russia

Bio and Neuro Inspired Computing and Applications (BIONCA 2021)

Nadia Nedjah

Luiza De Macedo Mourelle
State University of Rio de Janeiro, Brazil

State University of Rio de Janeiro, Brazil

Computational and Applied Mathematics (CAM 2021)

Maria Irene Falcão

University of Minho, Portugal

Fernando Miranda

University of Minho, Portugal

Computational and Applied Statistics (CAS 2021)

Ana Cristina Braga

University of Minho, Portugal

Computerized Evaluation of Economic Activities: Urban Spaces (CEEA 2021)

Diego Altafini

Università di Pisa, Italy

Valerio Cutini

Università di Pisa, Italy 
Computational Geometry and Applications (CGA 2021)

Marina Gavrilova

University of Calgary, Canada

Collaborative Intelligence in Multimodal Applications (CIMA 2021)

Robertas Damasevicius Kaunas University of Technology, Lithuania

Rytis Maskeliunas Kaunas University of Technology, Lithuania

Computational Optimization and Applications (COA 2021)

Ana Rocha

University of Minho, Portugal

Humberto Rocha

University of Coimbra, Portugal

\section{Computational Astrochemistry (CompAstro 2021)}

Marzio Rosi

Cecilia Ceccarelli

Stefano Falcinelli

Dimitrios Skouteris
University of Perugia, Italy

University of Grenoble, France

University of Perugia, Italy

Master-Up, Italy

\section{Computational Science and HPC (CSHPC 2021)}

Elise de Doncker

Fukuko Yuasa

Hideo Matsufuru
Western Michigan University, USA

High Energy Accelerator Research Organization (KEK), Japan

High Energy Accelerator Research Organization (KEK), Japan

\section{Cities, Technologies and Planning (CTP 2021)}

Malgorzata Hanzl

Beniamino Murgante

Ljiljana Zivkovic

Anastasia Stratigea

Giuseppe Borruso

Ginevra Balletto
University of Łódż, Poland

University of Basilicata, Italy

Ministry of Construction, Transport and Infrastructure/Institute of Architecture and Urban and Spatial Planning of Serbia, Serbia

National Technical University of Athens, Greece

University of Trieste, Italy

University of Cagliari, Italy

\section{Advanced Modeling E-Mobility in Urban Spaces (DEMOS 2021)}

Tiziana Campisi

Socrates Basbas

Ioannis Politis

Florin Nemtanu

Giovanna Acampa

Wolfgang Schulz
Kore University of Enna, Italy

Aristotle University of Thessaloniki, Greece

Aristotle University of Thessaloniki, Greece

Polytechnic University of Bucharest, Romania

Kore University of Enna, Italy

Zeppelin University, Germany 
Digital Transformation and Smart City (DIGISMART 2021)

Mauro Mazzei National Research Council, Italy

Econometric and Multidimensional Evaluation in Urban Environment (EMEUE 2021)

Carmelo Maria Torre Polytechnic University of Bari, Italy

Maria Cerreta

Pierluigi Morano

University "Federico II" of Naples, Italy

Simona Panaro

Polytechnic University of Bari, Italy

Francesco Tajani

University of Portsmouth, UK

Marco Locurcio

Sapienza University of Rome, Italy

Polytechnic University of Bari, Italy

The 11th International Workshop on Future Computing System Technologies and Applications (FiSTA 2021)

Bernady Apduhan

Rafael Santos
Kyushu Sangyo University, Japan

Brazilian National Institute for Space Research, Brazil

\section{Transformational Urban Mobility: Challenges and Opportunities During and Post COVID Era (FURTHER 2021)}

Tiziana Campisi

Socrates Basbas

Dilum Dissanayake

Kh Md Nahiduzzaman

Nurten Akgün Tanbay

Khaled J. Assi

Giovanni Tesoriere

Motasem Darwish
Kore University of Enna, Italy

Aristotle University of Thessaloniki, Greece

Newcastle University, UK

University of British Columbia, Canada

Bursa Technical University, Turkey

King Fahd University of Petroleum and Minerals, Saudi Arabia

Kore University of Enna, Italy

Middle East University, Jordan

Geodesign in Decision Making: Meta Planning and Collaborative Design for Sustainable and Inclusive Development (GDM 2021)

Francesco Scorza

Michele Campagna

Ana Clara Mourao Moura
University of Basilicata, Italy

University of Cagliari, Italy

Federal University of Minas Gerais, Brazil

Geomatics in Forestry and Agriculture: New Advances and Perspectives (GeoForAgr 2021)

Maurizio Pollino

Giuseppe Modica

Marco Vizzari
ENEA, Italy

University of Reggio Calabria, Italy

University of Perugia, Italy 
Geographical Analysis, Urban Modeling, Spatial Statistics (GEOG-AND-MOD 2021)

Beniamino Murgante University of Basilicata, Italy

Giuseppe Borruso University of Trieste, Italy

Hartmut Asche University of Potsdam, Germany

Geomatics for Resource Monitoring and Management (GRMM 2021)

Eufemia Tarantino

Enrico Borgogno Mondino

Alessandra Capolupo

Mirko Saponaro
Polytechnic University of Bari, Italy

University of Turin, Italy

Polytechnic University of Bari, Italy

Polytechnic University of Bari, Italy

12th International Symposium on Software Quality (ISSQ 2021)

Sanjay Misra

Covenant University, Nigeria

10th International Workshop on Collective, Massive and Evolutionary Systems (IWCES 2021)
Alfredo Milani
University of Perugia, Italy
Rajdeep Niyogi
Indian Institute of Technology, Roorkee, India

\section{Land Use Monitoring for Sustainability (LUMS 2021)}

$\begin{array}{ll}\text { Carmelo Maria Torre } & \text { Polytechnic University of Bari, Italy } \\ \text { Maria Cerreta } & \text { University "Federico II" of Naples, Italy } \\ \text { Massimiliano Bencardino } & \text { University of Salerno, Italy } \\ \text { Alessandro Bonifazi } & \text { Polytechnic University of Bari, Italy } \\ \text { Pasquale Balena } & \text { Polytechnic University of Bari, Italy } \\ \text { Giuliano Poli } & \text { University "Federico II" of Naples, Italy }\end{array}$

Machine Learning for Space and Earth Observation Data (MALSEOD 2021)

Rafael Santos

Karine Ferreira
Instituto Nacional de Pesquisas Espaciais, Brazil

Instituto Nacional de Pesquisas Espaciais, Brazil

Building Multi-dimensional Models for Assessing Complex Environmental Systems (MES 2021)

$\begin{array}{ll}\text { Marta Dell'Ovo } & \text { Polytechnic University of Milan, Italy } \\ \text { Vanessa Assumma } & \text { Polytechnic University of Turin, Italy } \\ \text { Caterina Caprioli } & \text { Polytechnic University of Turin, Italy } \\ \text { Giulia Datola } & \text { Polytechnic University of Turin, Italy } \\ \text { Federico dell'Anna } & \text { Polytechnic University of Turin, Italy }\end{array}$


Ecosystem Services: Nature's Contribution to People in Practice. Assessment Frameworks, Models, Mapping, and Implications (NC2P 2021)

Francesco Scorza

Sabrina Lai

Ana Clara Mourao Moura

Corrado Zoppi

Dani Broitman
University of Basilicata, Italy

University of Cagliari, Italy

Federal University of Minas Gerais, Brazil

University of Cagliari, Italy

Technion, Israel Institute of Technology, Israel

\section{Privacy in the Cloud/Edge/IoT World (PCEIoT 2021)}
Michele Mastroianni
University of Campania Luigi Vanvitelli, Italy
Lelio Campanile
Mauro Iacono
University of Campania Luigi Vanvitelli, Italy
University of Campania Luigi Vanvitelli, Italy
Processes, Methods and Tools Towards RESilient Cities and Cultural Heritage Prone to SOD and ROD Disasters (RES 2021)
Elena Cantatore
Alberico Sonnessa
Dario Esposito
Polytechnic University of Bari, Italy
Polytechnic University of Bari, Italy
Polytechnic University of Bari, Italy

Risk, Resilience and Sustainability in the Efficient Management of Water Resources: Approaches, Tools, Methodologies and Multidisciplinary Integrated Applications (RRS 2021)
Maria Macchiaroli
University of Salerno, Italy
Chiara D’Alpaos
Università degli Studi di Padova, Italy
Mirka Mobilia
Università degli Studi di Salerno, Italy
Antonia Longobardi
Università degli Studi di Salerno, Italy
Grazia Fattoruso
ENEA Research Center, Italy
Vincenzo Pellecchia
Ente Idrico Campano, Italy

\section{Scientific Computing Infrastructure (SCI 2021)}

Elena Stankova

Vladimir Korkhov

Natalia Kulabukhova
St. Petersburg University, Russia

St. Petersburg University, Russia

St. Petersburg University, Russia

\section{Smart Cities and User Data Management (SCIDAM 2021)}

Chiara Garau

Luigi Mundula

Gianni Fenu

Paolo Nesi

Paola Zamperlin
University of Cagliari, Italy University of Cagliari, Italy University of Cagliari, Italy University of Florence, Italy University of Pisa, Italy 
13th International Symposium on Software Engineering Processes and Applications (SEPA 2021)

Sanjay Misra

Covenant University, Nigeria

Ports of the Future - Smartness and Sustainability (SmartPorts 2021)

Patrizia Serra

Gianfranco Fancello

Ginevra Balletto

Luigi Mundula

Marco Mazzarino

Giuseppe Borruso

Maria del Mar Munoz

Leonisio
University of Cagliari, Italy

University of Cagliari, Italy

University of Cagliari, Italy

University of Cagliari, Italy

University of Venice, Italy

University of Trieste, Italy

Universidad de Cádiz, Spain

\section{Smart Tourism (SmartTourism 2021)}

Giuseppe Borruso

Silvia Battino

Ginevra Balletto

Maria del Mar Munoz

Leonisio

Ainhoa Amaro Garcia

Francesca Krasna
University of Trieste, Italy

University of Sassari, Italy

University of Cagliari, Italy

Universidad de Cádiz, Spain

Universidad de Alcalà/Universidad de Las Palmas, Spain

University of Trieste, Italy

Sustainability Performance Assessment: Models, Approaches and Applications toward Interdisciplinary and Integrated Solutions (SPA 2021)

Francesco Scorza

Sabrina Lai

Jolanta Dvarioniene

Valentin Grecu

Corrado Zoppi

Iole Cerminara
University of Basilicata, Italy

University of Cagliari, Italy

Kaunas University of Technology, Lithuania

Lucian Blaga University, Romania

University of Cagliari, Italy

University of Basilicata, Italy

Smart and Sustainable Island Communities (SSIC 2021)

Chiara Garau

Anastasia Stratigea

Paola Zamperlin

Francesco Scorza
University of Cagliari, Italy

National Technical University of Athens, Greece

University of Pisa, Italy

University of Basilicata, Italy

Science, Technologies and Policies to Innovate Spatial Planning (STP4P 2021)

Chiara Garau

Daniele La Rosa

Francesco Scorza
University of Cagliari, Italy

University of Catania, Italy

University of Basilicata, Italy 

Anna Maria Colavitti
University of Cagliari, Italy
Beniamino Murgante
University of Basilicata, Italy
Paolo La Greca
University of Catania, Italy

\section{Sustainable Urban Energy Systems (SURENSYS 2021)}

Luigi Mundula

University of Cagliari, Italy

Emilio Ghiani

University of Cagliari, Italy

\section{Space Syntax for Cities in Theory and Practice (Syntax_City 2021)}

Claudia Yamu

Akkelies van Nes

Chiara Garau
University of Groningen, The Netherlands

Western Norway University of Applied Sciences, Norway

University of Cagliari, Italy

Theoretical and Computational Chemistry and Its Applications (TCCMA 2021)

Noelia Faginas-Lago University of Perugia, Italy

13th International Workshop on Tools and Techniques in Software Development Process (TTSDP 2021)

Sanjay Misra

Covenant University, Nigeria

\section{Urban Form Studies (UForm 2021)}

Malgorzata Hanzl

Beniamino Murgante

Eufemia Tarantino

Irena Itova
Łódź University of Technology, Poland

University of Basilicata, Italy

Polytechnic University of Bari, Italy

University of Westminster, UK

\section{Urban Space Accessibility and Safety (USAS 2021)}

Chiara Garau

Francesco Pinna

Claudia Yamu

Vincenza Torrisi

Matteo Ignaccolo

Michela Tiboni

Silvia Rossetti
University of Cagliari, Italy

University of Cagliari, Italy

University of Groningen, The Netherlands

University of Catania, Italy

University of Catania, Italy

University of Brescia, Italy

University of Parma, Italy

\section{Virtual and Augmented Reality and Applications (VRA 2021)}
Osvaldo Gervasi
University of Perugia, Italy
Damiano Perri
University of Perugia, Italy
Marco Simonetti
University of Perugia, Italy
Sergio Tasso
University of Perugia, Italy 


\section{Workshop on Advanced and Computational Methods for Earth Science Applications (WACM4ES 2021)}

Luca Piroddi

Laura Foddis

Augusto Montisci

Sergio Vincenzo Calcina

Sebastiano D'Amico

Giovanni Martinelli
University of Cagliari, Italy

University of Cagliari, Italy

University of Cagliari, Italy

University of Cagliari, Italy

University of Malta, Malta

Istituto Nazionale di Geofisica e Vulcanologia, Italy/Chinese Academy of Sciences, China

\section{Sponsoring Organizations}

ICCSA 2021 would not have been possible without the tremendous support of many organizations and institutions, for which all organizers and participants of ICCSA 2021 express their sincere gratitude:

\section{包 Springer}
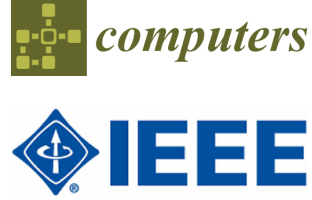

Italy Section

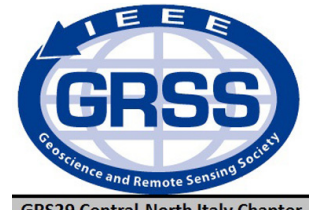

GRS29 Central-North Italy Chapter
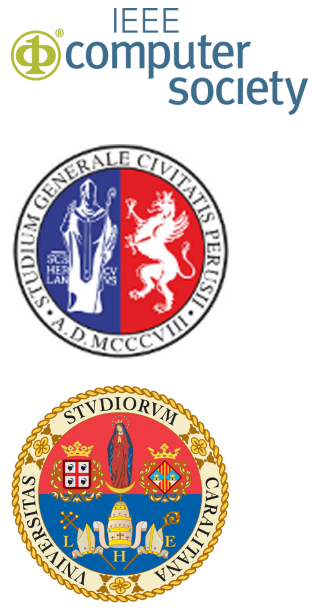

Springer International Publishing AG, Germany (https://www.springer.com)

Computers Open Access Journal

(https://www.mdpi.com/journal/computers)

IEEE Italy Section, Italy

(https://italy.ieeer8.org/)

Centre-North Italy Chapter IEEE GRSS, Italy (https://cispio.diet.uniroma1.it/marzano/ieee-grs/ index.html)

Italy Section of the Computer Society, Italy (https://site.ieee.org/italy-cs/)

University of Perugia, Italy

(https://www.unipg.it)

University of Cagliari, Italy

(https://unica.it/) 


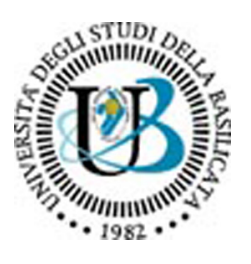

MONASH University

\section{K S U}

九州産業大学

KYUSHU SANGYO UNIVERSITY

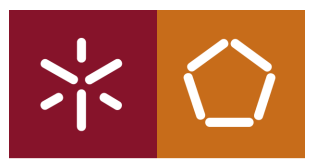

Universidade do Minho Escola de Engenharia
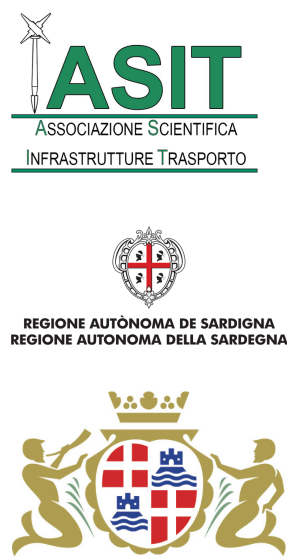

COMUNE DI CAGLIARI

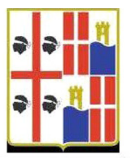

CITTÀ METROPOLITANA DI CAGLIARI

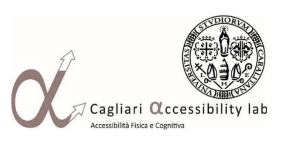

University of Basilicata, Italy

(http://www.unibas.it)

Monash University, Australia

(https://www.monash.edu/)

Kyushu Sangyo University, Japan

(https://www.kyusan-u.ac.jp/)

University of Minho, Portugal

(https://www.uminho.pt/)

Scientific Association Transport Infrastructures, Italy

(https://www.stradeeautostrade.it/associazioni-eorganizzazioni/asit-associazione-scientificainfrastrutture-trasporto/)

Regione Sardegna, Italy

(https://regione.sardegna.it/)

Comune di Cagliari, Italy

(https://www.comune.cagliari.it/)

Città Metropolitana di Cagliari

Cagliari Accessibility Lab (CAL)

(https://www.unica.it/unica/it/cagliari_ accessibility_lab.page/) 


\section{Referees}

Nicodemo Abate

Andre Ricardo Abed Grégio

Nasser Abu Zeid

Lidia Aceto

Nurten Akgün Tanbay

Filipe Alvelos

Paula Amaral

Federico Amato

Marina Alexandra Pedro Andrade

Debora Anelli

Alfonso Annunziata

Fahim Anzum

Tatsumi Aoyama

Bernady Apduhan

Jonathan Apeh

Vasilike Argyropoulos

Giuseppe Aronica

Daniela Ascenzi

Vanessa Assumma

Muhammad Attique Khan

Vecdi Aytaç

Alina Elena Baia

Ginevra Balletto

Marialaura Bancheri

Benedetto Barabino

Simona Barbaro

Enrico Barbierato

Jeniffer Barreto

Michele Bartalini

Socrates Basbas

Silvia Battino

Marcelo Becerra Rozas

Ranjan Kumar Behera

Emanuele Bellini

Massimo Bilancia

Giulio Biondi

Adriano Bisello

Ignacio Blanquer

Semen Bochkov

Alexander Bogdanov

Silvia Bonettini

Enrico Borgogno Mondino

Giuseppe Borruso
IMAA, National Research Council, Italy

Federal University of Paraná State, Brazil

Università di Ferrara, Italy

Università del Piemonte Orientale, Italy

Bursa Technical University, Turkey

Universidade do Minho, Portugal

Universidade Nova de Lisboa, Portugal

University of Lausanne, Switzerland

ISCTE-IUL, Portugal

Sapienza University of Rome, Italy

University of Cagliari, Italy

University of Calgary, Canada

High Energy Accelerator Research Organization, Japan

Kyushu Sangyo University, Japan

Covenant University, Nigeria

University of West Attica, Greece

Università di Messina, Italy

Università degli Studi di Trento, Italy

Politecnico di Torino, Italy

HITEC University Taxila, Pakistan

Ege University, Turkey

University of Perugia, Italy

University of Cagliari, Italy

ISAFOM, National Research Council, Italy

University of Brescia, Italy

Università degli Studi di Palermo, Italy

Università Cattolica del Sacro Cuore di Milano, Italy

Istituto Superior Técnico, Lisboa, Portugal

TAGES, Italy

Aristotle University of Thessaloniki, Greece

University of Sassari, Italy

Pontificia Universidad Católica de Valparaíso, Chile

National Institute of Technology, Rourkela, India

University of Campania Luigi Vanvitelli, Italy

University of Bari Aldo Moro, Italy

University of Firenze, Italy

Eurac Research, Italy

Universitat Politècnica de València, Spain

Ulyanovsk State Technical University, Russia

St. Petersburg University, Russia

University of Modena and Reggio Emilia, Italy

Università di Torino, Italy

University of Trieste, Italy 
Michele Bottazzi

Rahma Bouaziz

Ouafik Boulariah

Tulin Boyar

Ana Cristina Braga

Paolo Bragolusi

Luca Braidotti

Alessandro Buccini

Jorge Buele

Andrea Buffoni

Sergio Vincenzo Calcina

Michele Campagna

Lelio Campanile

Tiziana Campisi

Antonino Canale

Elena Cantatore

Pasquale Cantiello

Alessandra Capolupo

David Michele Cappelletti

Caterina Caprioli

Sara Carcangiu

Pedro Carrasqueira

Arcangelo Castiglione

Giulio Cavana

Davide Cerati

Maria Cerreta

Daniele Cesini

Jabed Chowdhury

Gennaro Ciccarelli

Birol Ciloglugil

Elena Cocuzza

Anna Maria Colavitt

Cecilia Coletti

Alberto Collu

Anna Concas

Mauro Coni

Melchiorre Contino

Antonella Cornelio

Aldina Correia

Elisete Correia

Florbela Correia

Stefano Corsi

Alberto Cortez

Lino Costa
University of Trento, Italy

Taibah University, Saudi Arabia

University of Salerno, Italy

Yildiz Technical University, Turkey

University of Minho, Portugal

University of Padova, Italy

University of Trieste, Italy

University of Cagliari, Italy

Universidad Tecnológica Indoamérica, Ecuador

TAGES, Italy

University of Cagliari, Italy

University of Cagliari, Italy

Università degli Studi della Campania Luigi Vanvitelli, Italy

Kore University of Enna, Italy

Kore University of Enna, Italy

DICATECh, Polytechnic University of Bari, Italy

Istituto Nazionale di Geofisica e Vulcanologia, Italy

Polytechnic University of Bari, Italy

University of Perugia, Italy

Politecnico di Torino, Italy

University of Cagliari, Italy

INESC Coimbra, Portugal

University of Salerno, Italy

Politecnico di Torino, Italy

Politecnico di Milano, Italy

University of Naples Federico II, Italy

INFN-CNAF, Italy

La Trobe University, Australia

Iuav University of Venice, Italy

Ege University, Turkey

Univesity of Catania, Italy

University of Cagliari, Italy

Università "G. d'Annunzio" di Chieti-Pescara, Italy

Independent Researcher, Italy

University of Basilicata, Italy

University of Cagliari, Italy

Università di Palermo, Italy

Università degli Studi di Brescia, Italy

Politécnico do Porto, Portugal

Universidade de Trás-os-Montes e Alto Douro, Portugal

Polytechnic Institute of Viana do Castelo, Portugal

Università degli Studi di Milano, Italy

Polytechnic of University Coimbra, Portugal

Universidade do Minho, Portugal 
Alessandro Costantini

Marilena Cozzolino

Giulia Crespi

Maurizio Crispino

Chiara D'Alpaos

Roberta D'Ambrosio

Sebastiano D'Amico

Hiroshi Daisaka

Gaia Daldanise

Robertas Damasevicius

Maria Danese

Bartoli Daniele

Motasem Darwish

Giulia Datola

Regina de Almeida

Elise de Doncker

Mariella De Fino

Giandomenico De Luca

Luiza de Macedo Mourelle

Gianluigi De Mare

Itamir de Morais Barroca Filho

Samuele De Petris

Marcilio de Souto

Alexander Degtyarev

Federico Dell'Anna

Marta Dell'Ovo

Fernanda Della Mura

Ahu Dereli Dursun

Bashir Derradji

Giulia Desogus

Marco Dettori

Frank Devai

Felicia Di Liddo

Valerio Di Pinto

Joana Dias

Luis Dias

Patricia Diaz de Alba

Isabel Dimas

Aleksandra Djordjevic

Luigi Dolores

Marco Donatelli

Doina Cristina Duma

Fabio Durastante

Aziz Dursun

Juan Enrique-Romero
INFN, Italy

Università del Molise, Italy

Politecnico di Torino, Italy

Politecnico di Milano, Italy

University of Padova, Italy

Università di Salerno, Italy

University of Malta, Malta

Hitotsubashi University, Japan

Italian National Research Council, Italy

Silesian University of Technology, Poland

ISPC, National Research Council, Italy

University of Perugia, Italy

Middle East University, Jordan

Politecnico di Torino, Italy

UTAD, Portugal

Western Michigan University, USA

Politecnico di Bari, Italy

Mediterranean University of Reggio Calabria, Italy

State University of Rio de Janeiro, Brazil

University of Salerno, Italy

Federal University of Rio Grande do Norte, Brazil

Università di Torino, Italy

LIFO, University of Orléans, France

St. Petersburg University, Russia

Politecnico di Torino, Italy

Politecnico di Milano, Italy

University of Naples "Federico II", Italy

Istanbul Commerce University, Turkey

University of Sfax, Tunisia

Università degli Studi di Cagliari, Italy

Università degli Studi di Sassari, Italy

London South Bank University, UK

Polytechnic University of Bari, Italy

University of Naples "Federico II", Italy

University of Coimbra, Portugal

University of Minho, Portugal

Gran Sasso Science Institute, Italy

University of Coimbra, Portugal

University of Belgrade, Serbia

Università degli Studi di Salerno, Italy

University of Insubria, Italy

INFN-CNAF, Italy

University of Pisa, Italy

Virginia Tech University, USA

Université Grenoble Alpes, France 
Annunziata Esposito

Amideo

Dario Esposito

Claudio Estatico

Noelia Faginas-Lago

Maria Irene Falcão

Stefano Falcinelli

Alessandro Farina

Grazia Fattoruso

Caterina Fenu

Luisa Fermo

Florbela Fernandes

Rosário Fernandes

Luis Fernandez-Sanz

Alessia Ferrari

Luís Ferrás

Ângela Ferreira

Flora Ferreira

Manuel Carlos Figueiredo

Ugo Fiore

Amedeo Flora

Hector Florez

Maria Laura Foddis

Valentina Franzoni

Adelaide Freitas

Samuel Frimpong

Ioannis Fyrogenis

Marika Gaballo

Laura Gabrielli

Ivan Gankevich

Chiara Garau

Ernesto Garcia Para

Fernando Garrido

Marina Gavrilova

Silvia Gazzola

Georgios Georgiadis

Osvaldo Gervasi

Andrea Gioia

Dario Gioia

Raffaele Giordano

Giacomo Giorgi

Eleonora Giovene

di Girasole

Salvatore Giuffrida

Marco Gola
University College Dublin, Ireland

Polytechnic University of Bari, Italy

University of Genova, Italy

Università di Perugia, Italy

University of Minho, Portugal

University of Perugia, Italy

University of Pisa, Italy

ENEA, Italy

University of Cagliari, Italy

University of Cagliari, Italy

Instituto Politecnico de Braganca, Portugal

University of Minho, Portugal

University of Alcala, Spain

Università di Parma, Italy

University of Minho, Portugal

Instituto Politécnico de Bragança, Portugal

University of Minho, Portugal

University of Minho, Portugal

University of Naples "Parthenope", Italy

University of Basilicata, Italy

Universidad Distrital Francisco Jose de Caldas, Colombia

University of Cagliari, Italy

Perugia University, Italy

University of Aveiro, Portugal

Durban University of Technology, South Africa

Aristotle University of Thessaloniki, Greece

Politecnico di Torino, Italy

Iuav University of Venice, Italy

St. Petersburg University, Russia

University of Cagliari, Italy

Universidad del País Vasco, Spain,

Universidad Técnica del Norte, Ecuador

University of Calgary, Canada

University of Bath, UK

Aristotle University of Thessaloniki, Greece

University of Perugia, Italy

Polytechnic University of Bari, Italy

ISPC-CNT, Italy

IRSS, National Research Council, Italy

University of Perugia, Italy

IRISS, National Research Council, Italy

Università di Catania, Italy

Politecnico di Milano, Italy 
A. Manuela Gonçalves Yuriy Gorbachev Angela Gorgoglione Yusuke Gotoh Anestis Gourgiotis Valery Grishkin Alessandro Grottesi Eduardo Guerra Ayse Giz Gulnerman Sevin Gümgüm Himanshu Gupta Sandra Haddad Malgorzata Hanzl Shoji Hashimoto Peter Hegedus Eligius M. T. Hendrix Edmond Ho Guan Yue Hong Vito Iacobellis Mauro Iacono Chiara Iacovino Antonino Iannuzzo Ali Idri Oana-Ramona Ilovan Mustafa Inceoglu Tadashi Ishikawa Federica Isola Irena Itova Edgar David de Izeppi Marija Jankovic Adrian Jaramillo Monalisa Jena Dorota Kamrowska-Załuska Issaku Kanamori Korhan Karabulut Yeliz Karaca Vicky Katsoni Dimitris Kavroudakis Shuhei Kimura Joanna Kolozej Vladimir Korkhov Thales Körting Tomonori Kouya Sylwia Krzysztofik Nataliia Kulabukhova Shrinivas B. Kulkarni
University of Minho, Portugal

Coddan Technologies LLC, Russia

Universidad de la República, Uruguay

Okayama University, Japan

University of Thessaly, Greece

St. Petersburg University, Russia

CINECA, Italy

Free University of Bozen-Bolzano, Italy

Ankara HBV University, Turkey

Izmir University of Economics, Turkey

BITS Pilani, Hyderabad, India

Arab Academy for Science, Egypt

Lodz University of Technology, Poland

KEK, Japan

University of Szeged, Hungary

Universidad de Málaga, Spain

Northumbria University, UK

Western Michigan University, USA

Polytechnic University of Bari, Italy

Università degli Studi della Campania, Italy

University of Basilicata, Italy

ETH Zurich, Switzerland

University Mohammed V, Morocco

Babeş-Bolyai University, Romania

Ege University, Turkey

KEK, Japan

University of Cagliari, Italy

University of Westminster, UK

VTTI, USA

CERTH, Greece

Universidad Tecnológica Metropolitana, Chile

Fakir Mohan University, India

Gdansk University of Technology, Poland

RIKEN Center for Computational Science, Japan

Yasar University, Turkey

University of Massachusetts Medical School, USA

University of West Attica, Greece

University of the Aegean, Greece

Okayama University, Japan

Cracow University of Technology, Poland

St. Petersburg University, Russia

INPE, Brazil

Shizuoka Institute of Science and Technology, Japan Lodz University of Technology, Poland

St. Petersburg University, Russia

SDM College of Engineering and Technology, India 
Pavan Kumar

Anisha Kumari

Ludovica La Rocca

Daniele La Rosa

Sabrina Lai

Giuseppe Francesco Cesare Lama

Mariusz Lamprecht

Vincenzo Laporta

Chien-Sing Lee

José Isaac Lemus Romani

Federica Leone

Alexander H. Levis

Carola Lingua

Marco Locurcio

Andrea Lombardi

Savino Longo

Fernando Lopez Gayarre

Yan Lu

Maria Macchiaroli

Helmuth Malonek

Francesca Maltinti

Luca Mancini

Marcos Mandado

Ernesto Marcheggiani

Krassimir Markov

Giovanni Martinelli

Alessandro Marucci

Fiammetta Marulli

Gabriella Maselli

Rytis Maskeliunas

Michele Mastroianni

Cristian Mateos

Hideo Matsufuru

D’Apuzzo Mauro

Chiara Mazzarella

Marco Mazzarino

Giovanni Mei

Mário Melo

Francesco Mercaldo

Alfredo Milani

Alessandra Milesi

Antonio Minervino

Fernando Miranda
University of Calgary, Canada

National Institute of Technology, Rourkela, India

University of Naples "Federico II", Italy

University of Catania, Italy

University of Cagliari, Italy

University of Naples "Federico II", Italy

University of Lodz, Poland

National Research Council, Italy

Sunway University, Malaysia

Pontifical Catholic University of Valparaíso, Chile

University of Cagliari, Italy

George Mason University, USA

Polytechnic University of Turin, Italy

Polytechnic University of Bari, Italy

University of Perugia, Italy

University of Bari, Italy

University of Oviedo, Spain

Western Michigan University, USA

University of Salerno, Italy

University of Aveiro, Portugal

University of Cagliari, Italy

University of Perugia, Italy

University of Vigo, Spain

Università Politecnica delle Marche, Italy

University of Telecommunications and Post, Bulgaria

INGV, Italy

University of L'Aquila, Italy

University of Campania Luigi Vanvitelli, Italy

University of Salerno, Italy

Kaunas University of Technology, Lithuania

University of Campania Luigi Vanvitelli, Italy

Universidad Nacional del Centro de la Provincia de Buenos Aires, Argentina

High Energy Accelerator Research Organization (KEK), Japan

University of Cassino and Southern Lazio, Italy

University Federico II, Italy

University of Venice, Italy

University of Cagliari, Italy

Federal Institute of Rio Grande do Norte, Brazil

University of Molise, Italy

University of Perugia, Italy

University of Cagliari, Italy

ISPC, National Research Council, Italy

Universidade do Minho, Portugal 
B. Mishra

Sanjay Misra

Mirka Mobilia

Giuseppe Modica

Mohammadsadegh

Mohagheghi

Mohamad Molaei Qelichi

Mario Molinara

Augusto Montisci

Pierluigi Morano

Ricardo Moura

Ana Clara Mourao Moura

Maria Mourao

Daichi Mukunoki

Beniamino Murgante

Naohito Nakasato

Grazia Napoli

Isabel Cristina Natário

Nadia Nedjah

Antonio Nesticò

Andreas Nikiforiadis

Keigo Nitadori

Silvio Nocera

Giuseppina Oliva

Arogundade Oluwasefunmi

Ken-ichi Oohara

Tommaso Orusa

M. Fernanda P. Costa

Roberta Padulano

Maria Panagiotopoulou

Jay Pancham

Gianni Pantaleo

Dimos Pantazis

Michela Paolucci

Eric Pardede

Olivier Parisot

Vincenzo Pellecchia

Anna Pelosi

Edit Pengő

Marco Pepe

Paola Perchinunno

Ana Pereira

Mariano Pernetti

Damiano Perri
University of Szeged, Hungary

Covenant University, Nigeria

University of Salerno, Italy

Università degli Studi di Reggio Calabria, Italy

Vali-e-Asr University of Rafsanjan, Iran

University of Tehran, Iran

University of Cassino and Southern Lazio, Italy

Università degli Studi di Cagliari, Italy

Polytechnic University of Bari, Italy

Universidade Nova de Lisboa, Portugal

Federal University of Minas Gerais, Brazil

Polytechnic Institute of Viana do Castelo, Portugal

RIKEN Center for Computational Science, Japan

University of Basilicata, Italy

University of Aizu, Japan

Università degli Studi di Palermo, Italy

Universidade Nova de Lisboa, Portugal

State University of Rio de Janeiro, Brazil

University of Salerno, Italy

Aristotle University of Thessaloniki, Greece

RIKEN Center for Computational Science, Japan

Iuav University of Venice, Italy

University of Salerno, Italy

Academy of Mathematics and System Science, China

University of Tokyo, Japan

University of Turin, Italy

University of Minho, Portugal

Centro Euro-Mediterraneo sui Cambiamenti Climatici, Italy

National Technical University of Athens, Greece

Durban University of Technology, South Africa

University of Florence, Italy

University of West Attica, Greece

University of Florence, Italy

La Trobe University, Australia

Luxembourg Institute of Science and Technology, Luxembourg

Ente Idrico Campano, Italy

University of Salerno, Italy

University of Szeged, Hungary

University of Salerno, Italy

University of Cagliari, Italy

Polytechnic Institute of Bragança, Portugal

University of Campania, Italy

University of Perugia, Italy 


Federica Pes
Marco Petrelli
Massimiliano Petri
Khiem Phan
Alberto Ferruccio Piccinni
Angela Pilogallo
Francesco Pinna
Telmo Pinto
Luca Piroddi
Darius Plonis
Giuliano Poli
Maria João Polidoro
Ioannis Politis
Maurizio Pollino
Antonio Pratelli
Salvatore Praticò
Marco Prato
Carlotta Quagliolo
Emanuela Quaquero
Garrisi Raffaele
Nicoletta Rassu
Hafiz Tayyab Rauf
Michela Ravanelli
Roberta Ravanelli
Alfredo Reder

Stefania Regalbuto

Rommel Regis

Lothar Reichel

Marco Reis

Maria Reitano

Jerzy Respondek

Elisa Riccietti

Albert Rimola

Angela Rizzo

Ana Maria A. C. Rocha

Fabio Rocha

Humberto Rocha

Maria Clara Rocha

Miguel Rocha

Giuseppe Rodriguez

Guillermo Rodriguez

Elisabetta Ronchieri

Marzio Rosi

Silvia Rossetti

Marco Rossitti
University of Cagliari, Italy

Roma Tre University, Italy

University of Pisa, Italy

Duy Tan University, Vietnam

Polytechnic of Bari, Italy

University of Basilicata, Italy

University of Cagliari, Italy

University of Coimbra, Portugal

University of Cagliari, Italy

Vilnius Gediminas Technical University, Lithuania

University of Naples "Federico II", Italy

Polytecnic Institute of Porto, Portugal

Aristotle University of Thessaloniki, Greece

ENEA, Italy

University of Pisa, Italy

Mediterranean University of Reggio Calabria, Italy

University of Modena and Reggio Emilia, Italy

Polytechnic University of Turin, Italy

Univesity of Cagliari, Italy

Polizia postale e delle Comunicazioni, Italy

University of Cagliari, Italy

University of Bradford, UK

Sapienza University of Rome, Italy

Sapienza University of Rome, Italy

Centro Euro-Mediterraneo sui Cambiamenti Climatici, Italy

University of Naples "Federico II", Italy

Saint Joseph's University, USA

Kent State University, USA

University of Coimbra, Portugal

University of Naples "Federico II", Italy

Silesian University of Technology, Poland

École Normale Supérieure de Lyon, France

Universitat Autònoma de Barcelona, Spain

University of Bari, Italy

University of Minho, Portugal

Institute of Technology and Research, Brazil

University of Coimbra, Portugal

Polytechnic Institute of Coimbra, Portugal

University of Minho, Portugal

University of Cagliari, Italy

UNICEN, Argentina

INFN, Italy

University of Perugia, Italy

University of Parma, Italy

Polytechnic University of Milan, Italy 
Francesco Rotondo

Irene Rubino

Agustín Salas

Juan Pablo Sandoval Alcocer

Luigi Santopietro

Rafael Santos

Valentino Santucci

Mirko Saponaro

Filippo Sarvia

Marco Scaioni

Rafal Scherer

Francesco Scorza

Ester Scotto di Perta

Monica Sebillo

Patrizia Serra

Ricardo Severino

Jie Shen

Huahao Shou

Miltiadis Siavvas

Brandon Sieu

Ângela Silva

Carina Silva

Joao Carlos Silva

Fabio Silveira

Marco Simonetti

Ana Jacinta Soares

Maria Joana Soares

Michel Soares

George Somarakis

Maria Somma

Alberico Sonnessa

Elena Stankova

Flavio Stochino

Anastasia Stratigea

Yasuaki Sumida

Yue Sun

Kirill Sviatov

Daisuke Takahashi

Aladics Tamás

David Taniar

Rodrigo Tapia McClung

Eufemia Tarantino
Marche Polytechnic University, Italy

Polytechnic University of Turin, Italy

Pontifical Catholic University of Valparaíso, Chile

Universidad Católica Boliviana "San Pablo", Bolivia

University of Basilicata, Italy

National Institute for Space Research, Brazil

Università per Stranieri di Perugia, Italy

Polytechnic University of Bari, Italy

University of Turin, Italy

Polytechnic University of Milan, Italy

Częstochowa University of Technology, Poland

University of Basilicata, Italy

University of Napoli "Federico II", Italy

University of Salerno, Italy

University of Cagliari, Italy

University of Minho, Portugal

University of Michigan, USA

Zhejiang University of Technology, China

Centre for Research and Technology Hellas, Greece

University of Calgary, Canada

Instituto Politécnico de Viana do Castelo, Portugal

Polytechic Institute of Lisbon, Portugal

Polytechnic Institute of Cavado and Ave, Portugal

Federal University of Sao Paulo, Brazil

University of Florence, Italy

University of Minho, Portugal

University of Minho, Portugal

Federal University of Sergipe, Brazil

Foundation for Research and Technology Hellas, Greece

University of Naples "Federico II", Italy

Polytechnic University of Bari, Italy

St. Petersburg University, Russia

University of Cagliari, Italy

National Technical University of Athens, Greece

Kyushu Sangyo University, Japan

European X-Ray Free-Electron Laser Facility, Germany

Ulyanovsk State Technical University, Russia

University of Tsukuba, Japan

University of Szeged, Hungary

Monash University, Australia

Centro de Investigación en Ciencias de Información Geoespacial, Mexico

Polytechnic University of Bari, Italy 
Sergio Tasso

Ana Paula Teixeira

Senhorinha Teixeira

Tengku Adil Tengku Izhar

Maria Filomena Teodoro

Giovanni Tesoriere

Yiota Theodora

Graça Tomaz

Carmelo Maria Torre

Francesca Torrieri

Vincenza Torrisi

Vincenzo Totaro

Pham Trung

Dimitrios Tsoukalas

Sanjida Tumpa

Iñaki Tuñon

Takahiro Ueda

Piero Ugliengo

Abdi Usman

Ettore Valente

Jordi Vallverdu

Cornelis Van Der Mee

José Varela-Aldás

Fanny Vazart

Franco Vecchiocattivi

Laura Verde

Giulia Vergerio

Jos Vermaseren

Giacomo Viccione

Marco Vizzari

Corrado Vizzarri

Alexander Vodyaho

Nikolay N. Voit

Marco Vona

Agustinus Borgy Waluyo

Fernando Wanderley

Chao Wang

Marcin Wozniak

Tiang Xian

Rekha Yadav

Claudia Yamu

Fenghui Yao
University of Perugia, Italy

Universidade de Trás-os-Montes e Alto Douro, Portugal

University of Minho, Portugal

Universiti Teknologi MARA, Malaysia

University of Lisbon/Portuguese Naval Academy, Portugal

Kore University of Enna, Italy

National Technical Univeristy of Athens, Greece

Polytechnic Institute of Guarda, Portugal

Polytechnic University of Bari, Italy

University of Naples "Federico II", Italy

University of Catania, Italy

Polytechnic University of Bari, Italy

Ho Chi Minh City University of Technology, Vietnam

Centre of Research and Technology Hellas (CERTH), Greece

University of Calgary, Canada

Universidad de Valencia, Spain

Seikei University, Japan

University of Turin, Italy

Haramaya University, Ethiopia

University of Naples "Federico II", Italy

Universitat Autònoma de Barcelona, Spain

University of Cagliari, Italy

Universidad Tecnológica Indoamérica, Ecuador

University of Grenoble Alpes, France

University of Perugia, Italy

University of Campania Luigi Vanvitelli, Italy

Polytechnic University of Turin, Italy

Nikhef, The Netherlands

University of Salerno, Italy

University of Perugia, Italy

Polytechnic University of Bari, Italy

St. Petersburg State Electrotechnical University "LETI", Russia

Ulyanovsk State Technical University, Russia

University of Basilicata, Italy

Monash University, Australia

Catholic University of Pernambuco, Brazil

University of Science and Technology of China, China

Silesian University of Technology, Poland

Nathong University, China

KL University, India

University of Groningen, The Netherlands

Tennessee State University, USA 
xxxiv Organization

Fukuko Yuasa

Moayid Ali Zaidi

Paola Zamperlin

Peter Zeile

Milliam Maxime Zekeng Ndadji

Nataly Zhukova

Ljiljiana Zivkovic
KEK, Japan

Ostfold University College Norway, Norway

University of Pisa, Italy

Karlsruhe Institute of Technology, Germany

University of Dschang, Cameroon

ITMO University, Russia

Ministry of Construction, Transport and Infrastructure/Institute of Architecture and Urban and Spatial Planning of Serbia, Serbia 


\section{Contents - Part VI}

\section{International Workshop on Digital Transformation and Smart City (DIGISMART 2021)}

Analysis of Regional Imbalances in Italy Based on Cluster Analysis . . . . . . .

Massimo De Maria, Mauro Mazzei, Oleg V. Bik, and Armando L. Palma

New Smart Mobility Applications: Preliminary Findings on a Pilot Study in the Municipality of Artena . . . . . . . . . . . . . . . . . . .

Mauro D’Apuzzo, Azzurra Evangelisti, Daniela Santilli, Stefano Buzzi, Mauro Mazzei, and Viviana Bietoni

\section{International Workshop on Econometrics and Multidimensional Evaluation in Urban Environment (EMEUE 2021)}

The Benefit Transfer Method for the Economic Evaluation of Urban Forests . . . . . . . . . . . . . . . . . . . . . . . . . . . . . .

Francesco Sica and Antonio Nesticò

The Effects of Covid-19 Pandemic on the Housing Market: A Case Study in Rome (Italy) . . . . . . . . . . . . . . . . . . . . . . . . . . . . . . . . . . . . .

Francesco Tajani, Pierluigi Morano, Felicia Di Liddo,

Maria Rosaria Guarini, and Rossana Ranieri

The Contribution of the Most Influencing Factors on the Housing Rents:

An Analysis in the City of Milan (Italy) . . . . . . . . . . . . .

Pierluigi Morano, Francesco Tajani, Felicia Di Liddo, Rossana Ranieri, and Paola Amoruso

The Paradox of Fiscal Inequality in Italy: Exploratory Analyses on Property

Tax Rates. . . . . . . . . . . . . . . . . . . . . . . . . . . . .

Rocco Curto, Alice Barreca, Giorgia Malavasi, and Diana Rolando

The Financial Costs in Energy Efficient District. Alternative Scenarios from the Demo Sites of the CITyFiED Program . . . . . . . . . . .

Simona Barbaro and Grazia Napoli

Inclusive Strategic Programming: Methodological Aspects of the Case Study of the Jonian Valleys of Peloritani (Sicily, Italy) . . . . . . . . . . . . .

Giuseppe Bombino, Francesco Calabrò, Giuseppina Cassalia, Lidia Errante, and Viviana Vinci 
New Housing Preferences in the COVID-19 Era: A Best-to-Worst

Scaling Experiment . . . . . . . . . . . . . . . . . . .

Marta Bottero, Marina Bravi, Caterina Caprioli, Federico Dell'Anna, Marta Dell'Ovo, and Alessandra Oppio

An Analysis of the Methods Applied for the Assessment of the Market

Value of Residential Properties in Italian Judicial Procedures . . . . . . . . . . .

Francesco Tajani, Felicia Di Liddo, Paola Amoruso, Francesco Sica, and Ivana La Spina

Integrated Statistical Data for Planning Social Housing in the City

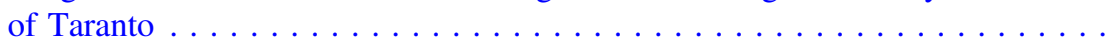

Paola Perchinunno and Francesco Rotondo

Reconstruction as an Opportunity to Promote Local Self-sustainable

Development of Shrinking Territories in Seismic Inner Areas

in Central Italy . . . . . . . . . . . . . . . . . . . . . . . . .

Luca Domenella, Marco Galasso, Giovanni Marinelli,

and Francesco Rotondo

Urban Regeneration Processes and Social Impact: A Literature Review

to Explore the Role of Evaluation . . . . . . . . . . . . . . . . . .

Maria Cerreta and Ludovica La Rocca

Using Artificial Neural Networks to Uncover Real Estate Market

Transparency: The Market Value . . . . . . . . . . . . . . . . . . . .

Laura Gabrielli, Aurora Greta Ruggeri, and Massimiliano Scarpa

Creative Ecosystem Services: Valuing Benefits of Innovative

Cultural Networks. . . . . . . . . . . . . . . . . . . . .

Giuliano Poli and Gaia Daldanise

Ecosystem Services and Land Take. A Composite Indicator for the Assessment of Sustainable Urban Projects . . . . . . . . . . . . . . .

Pierluigi Morano, Maria Rosaria Guarini, Francesco Sica, and Debora Anelli

Building Industry and Energy Efficiency: A Review of Three Major Issues at Stake . . . . . . . . . . . . . . . . . . . . . . . .

Sergio Copiello, Laura Gabrielli, and Ezio Micelli

An Evaluation Model for the Optimization of Property Sales

in Auction Markets . . . . . . . . . . . . . . . . . . . . . .

Francesco Tajani, Pierluigi Morano, Marco Locurcio, Paola Amoruso, and Carmelo Maria Torre 
Urban Transformation Interventions: A Decision Support Model for a Fair Rent Gap Recapture . . . . . . . . . . . . . . . . . . . . . . .

Pierluigi Morano, Francesco Tajani, Vincenzo del Giudice,

Pierfrancesco De Paola, and Debora Anelli

An Optimization Model for Supporting the Property Asset Allocation

Decision-Making Process . . . . . . . . . . . . . . . . . .

Francesco Tajani, Marco Locurcio, Pierluigi Morano,

and Debora Anelli

The Risks Assessment in the Project Financing Initiative for the Cemetery

Expansion Intervention in a Small Town in Southern Italy . . . . . . . . . . .

Marco Locurcio, Pierluigi Morano, Francesco Tajani, Felicia Di Liddo, and Carmelo Maria Torre

A Citizen-Led Spatial Information System for Collaborative (Post-) pandemic Urban Strategies: The Ponticelli Experience, Naples (Italy) . . . . . .

Maria Cerreta, Luigi Liccardi, and Maria Reitano

The Knowledge Phase of the Strategic Programming: The Case Study of the Jonian Valleys of Peloritani (Sicily, Italy) . . . . . . . . . . . . . . .

Giuseppe Bombino, Francesco Calabrò, Giuseppina Cassalia,

Lidia Errante, and Viviana Vinci

\section{International Workshop on Transformational Urban Mobility: Challenges and Opportunities During and Post COVID Era (FURTHER 2021)}

Developing Flexible Mobility On-Demand in the Era of Mobility as a Service: An Overview of the Italian Context Before and After Pandemic. . . . . . . . . . . . . . . . . . .

Tiziana Campisi, Chiara Garau, Giovanna Acampa, Francesca Maltinti, Antonino Canale, and Mauro Coni

Factors Influencing Public Transport Demand in Sicily During COVID-19 Era: A Study of Commuters' Travel and Mode Choice Behaviors . . . . . . . . .

Socrates Basbas, Georgios Georgiadis, Tiziana Campisi, and Giovanni Tesoriere

Standard Cost of Local Public Transport in the Post-COVID-19 Era:

The Italian Case . . . . . . . . . . . . . . . . . . . . . . . .

G. Acampa, M. Grasso, C. M. Parisi, D. Ticali, and A. Severino

COVID-19's Effects over E-commerce: A Preliminary Statistical Assessment for Some European Countries . . . . . . . . . . . . . . . .

Tiziana Campisi, Antonio Russo, Giovanni Tesoriere,

Efstathios Bouhouras, and Socrates Basbas 
The Impact of COVID-19 Pandemic on the Perception of Public

Transportation Users in Amman (Jordan) . . . . . . . . . . . . . . . . . .

Motasem Darwish, Tiziana Campisi, and Ghaida Abu Rumman

\section{International Workshop on Geodesign in Decision Making: Meta Planning and Collaborative Design for Sustainable and Inclusive Development (GDM 2021)}

Landscape Information Modelling to Improve Feedback in the Geodesign International Collaboration for Carbon Credit Enhancement in Metropolitan Regions - The Case Study of Fortaleza, Brazil . . . . . . . . . . . . . . . Newton Moura, Joana Guedes, Emiliano Cavalcante, Morganna Oliveira, Ana Maia, Anne Castro, Eugênio Moreira, Daniel Cardoso, and Vitor Sampaio

Decision Making and Geodesign: A Collaborative Territorial Planning Proposal for the Metropolitan Region of Belém, Pará, Brazil . . . . . . . . . . Alan Nunes Araújo, Tiago Barreto de Andrade Costa, Bruno Daniel das Neves Benitez, Fabricio Martins Silva, and Joabi Luiz Lima De Lima

Geodesign Applied to Propositional Scenarios of Medium and Long-Term

Sustainable Projects for Rio de Janeiro Metropolitan Region, Brazil . . . . . . . Tiago Badre Marino, Cézar Augusto Barra Rocha, Ashiley Adelaide Rosa, and Tiago Augusto Gonçalves Mello

Geodesign Using GISColab Platform: SDI Consumed by WMS and WFS \& WPS Protocols in Transformative-Learning Actions in Planning . . . . . . . . Ana Clara Mourão Moura, Christian Rezende Freitas, Vanessa Tenuta de Freitas, and Ana Isabel Anastasia de Sa

Geodesign Brazil: Trees for the Metropolitan Area of São Paulo. . . . . . . . . . 463 Adriana Afonso Sandre, Amanda Lombardo Fruehauf, Augusto Akio Lucchezi Miyahara, Ashiley Adelaide Rosa, Cíntia Miua Maruyama, Giuliano Maselli Locoselli, Leticia Figueiredo Candido, Magda Adelaide Lombardo, Matheus Aguiar Coelho, Rafael Pollastrini Murolo, Riciane Maria Reis Pombo, Taícia Helena Negrin Marques, and Paulo Renato Mesquita Pellegrino

The Potential of Geodesign for the Optimization of Land Use in the Perspective of Sustainability: Case Study of the Metropolitan Region of Campinas .........................

Andréia Medinilha Pancher, Ana Isabel de Sá, Marcelo Costa, and Tiago Oyan Aguiar 
Using Geodesign to Plan the Future of Macapa Metropolitan Region,

State of Amapa, Brazil: A Support to Expanding Collaborative

Technical Performance. . . . . . . . . . . . . . . . . . . . . . .

Gustavo Adolfo Tinoco Martínez, Fabiana Carmo de Vargas Vieira,

Caroline Cristiane Rocha, Ana Corina Maia Palheta, and Sara Heloiza Alberto Neri

Asynchronous Mode in the Webgis: A Challenge to Ensure Greater

Popular Participation . . . . . . . . . . . . . . . . . . . . .

Patricia PortoCarreiro, Patricia Vieira Trinta, and Thiago Lima e Lima

\section{1th International Workshop on Future Computing System Technologies and Applications (FiSTA 2021)}

Deep Fake Recognition in Tweets Using Text Augmentation,

Word Embeddings and Deep Learning.

Senait G. Tesfagergish, Robertas Damaševičius, and Jurgita Kapočiūté-Dzikiené

Development of an RL-Based Mechanism to Augment Computation Offloading in Edge Computing . . . . . . . . . . . . . . . . .

Shintaro Ide and Bernady O. Apduhan

An Initial Assessment of a Chatbot for Rumination-Focused Cognitive

Behavioral Therapy (RFCBT) in College Students. . . . . . . . . . . . . . . . .

Alana Lucia Souza Oliveira, Leonardo Nogueira Matos,

Methanias Colaço Junior, and Zenith Nara Costa Delabrida

Price Forecasting with Deep Learning in Business to Consumer Markets . . . .

Emre Eğriboz and Mehmet S. Aktaş

Modeling and Verification of Contactless Mobile Banking System in E-Banking Using SPIN . . . . . . . . . . . . . . . . . . . . . . . .

Tej Narayan Thakur and Noriaki Yoshiura

\section{International Workshop on Geographical Analysis, Urban Modeling, Spatial Statistics (GEOG-AND-MOD 2021)}

Earthquake Prediction Based on Combined Seismic and GPS

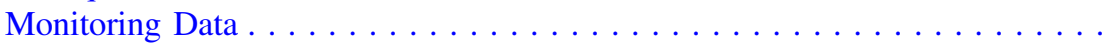

V. G. Gitis, A. B. Derendyaev, and K. N. Petrov

Survey of a Peruvian Archaeological Site Using LiDAR and Photogrammetry: A Contribution to the Study of the Chachapoya . . . . . . . . .

Giovanni Righetti, Stefano Serafini, Fabian Brondi Rueda,

Warren B. Church, and Gabriele Garnero 
Estimation of Hourly Salinity Concentrations Using an Artificial

Neural Network . . . . . . . . . . . . . . . . . . . . . .

Vladimir J. Alarcon, Anna C. Linhoss, Christopher R. Kelble,

Paul F. Mickle, Joseph Bishop, and Emily Milton

Tracing and Modeling of the COVID-19 Pandemic Infections in Poland

Using Spatial Interactions Models . . . . . . . . . . . . . . . .

Piotr A. Werner

On Sustainability of Urban Italian Mobility . . . . . . . . . . . . . . . . 658

Gabriella Schoier, Giuseppe Borruso, and Beatrice Dedemo

A Remote Sensing and Geo-Statistical Approaches to Mapping Burn

Areas in Apulia Region (Southern Italy) . . . . . . . . . . . . . . . . .

Valentina Santarsiero, Gabriele Nolè, Antonio Lanorte, Biagio Tucci,

Francesco Vito Ronco, Vito Augusto Capurso, and Beniamino Murgante

Soil Erosion and Land Degradation in Rural Environment: A Preliminary

GIS and Remote-Sensed Approach . . . . . . . . . . . . . . . . . . . . .

Giuseppe Cillis, Gabriele Nolè, Antonio Lanorte, Valentina Santarsiero,

Biagio Tucci, Francesco Scorza, and Beniamino Murgante

A Remote Sensing Methodology to Assess the Abandoned Arable Land

Using NDVI Index in Basilicata Region. . . . . . . . . . . . . . . . . . .

Valentina Santarsiero, Gabriele Nolè, Antonio Lanorte, Biagio Tucci,

Giuseppe Cillis, Francesco Scorza, and Beniamino Murgante

Assessment and Monitoring of Soil Erosion Risk and Land Degradation in Arable Land Combining Remote Sensing Methodologies

and RUSLE Factors. . . . . . . . . . . . . . . . . . . . . . . . .

Biagio Tucci, Gabriele Nolè, Antonio Lanorte, Valentina Santarsiero,

Giuseppe Cillis, Francesco Scorza, and Beniamino Murgante

Author Index 


\title{
The Risks Assessment in the Project Financing Initiative for the Cemetery Expansion Intervention in a Small Town in Southern Italy
}

\author{
Marco Locurcio $^{1}$, Pierluigi Morano ${ }^{1}$, Francesco Tajani ${ }^{2}$, Felicia Di Liddo $^{1(\otimes)}$, \\ and Carmelo Maria Torre ${ }^{1}$ \\ ${ }^{1}$ Department of Civil, Environmental, Land, Building Engineering and Chemistry, Polytechnic \\ University of Bari, 70126 Bari, Italy \\ felicia.diliddo@poliba.it \\ 2 Department of Architecture and Design, "Sapienza" University of Rome, 00196 Rome, Italy
}

\begin{abstract}
In the present research the risks matrix related to a transformation intervention to be carried out through the Project Financing (PF) operational tool, has been developed. With reference to the expansion and management of the cemetery of a small town located in Southern Italy, the identification and allocation of the risks among the parties involved - private investor and Public Administration - have been implemented. Furthermore, the verification of the feasibility by the Public Administration in the use of the PF operational tool has been performed, by analyzing the results obtained by the project proposer subject in financial terms. The risk assessment constitutes a support tool for the public Administration in the decision-making processes aimed to evaluate the PF proposals by the proposer subject in order to ensure an appropriate and detailed investigation on the Public Private Partnerships mechanism and to avoid complications and contingencies that could lead to initiative failure. In this sense, the present analysis allows to evaluate the advantages for public and private subjects to use the PF mechanism in bridging the existing gulf between the scarce public resources and the investment demand of expansion and/or redevelopment of urban cemeteries.
\end{abstract}

Keywords: Public-Private Partnership · Project Financing · Expansion intervention - Municipal cemeteries - New realization project - Small town · Risks matrix $\cdot$ Business plan

\section{Introduction}

In the context of the Public Private Partnerships (PPP), the Project Financing (PF) constitutes an increasingly being used operational tool for regions and municipalities able to bridge the gap between the available financial resources and the investment needs for the realization and/or redevelopment of infrastructures and collective services [1-3, 11]. Moreover, the PF allows to channel the efficiency and the qualitative standard of the private sector in the renovation of public interest properties and abandoned areas located on urban territory. 
In general terms, the PF represents a complex procedure in which different fields are involved - legislative, economic and financial -. The Public Administration may use it in order to carry out projects with great technical difficulties and high capital requirement. The PF, in fact, is a contractual tool intended to ensure the cooperation between public entities and private investors in the context of initiative for the territorial development [16].

The satisfaction of the public interest deriving from the realization of the infrastructure is verified without direct public financial burdens. Thus, the central feature of the PF mechanism concerns the financial backing of the planned investments by the private investor, i.e. the design phase and the realization, in exchange for the project direct or indirect management.

Therefore, the financing procedure is not adaptable to all initiatives that require high investments, but only to those able of generating profits, in order to ensure the positive financial balance for the private investor in terms of profit deriving from the cash flows related to the intervention higher than the zero value.

In general, in PPP procedures the public subject has to assess not exclusively the public interest related to the proposal, but to verify its financial feasibility to include the project in the interventions to be realized planning [14].

In the context of the cemetery construction, the PF has over time become a procedural tool implemented by Public Administration for realization and management of cemetery areas, especially due to the convenience in financial and risks terms [8, 17].

The progressive involvement of private sector in projects related to cemetery spaces attests the growing interest in the PF able to allow also the small towns' public entities, unable to bear the required costs for the renovate or expansion of cemeteries, to outsource the redevelopment and management of these.

In the Italian context, the dimensions reached by PF market in the cemetery sector in terms of number of started interventions and activated investments are significant i.e. among the most relevant are mentioned the projects realized in the cities of Foligno (2003), Avellino (2005), Taranto (2004), Castel Maggiore (2005), Sassari (2007), Pescara (2007), Messina (2008), Latina (2007), Potenza (2009), Venosa (2009), etc. [5].

Through the PF contractual mechanism, the public subjects could obtain a savings in economic and administrative terms. During the concession period, in fact, the Public Administration monitors the agreement performance, as it does not be directly concerned with the niches construction and management. Furthermore, the long-term duration of the public private cooperation allows the Public Administration to avoid the congestion risk of cemeteries and, consequently, to prevent sanitary criticality associated to it. From the private point of view, the interest in PF projects regards the low construction and management costs, the certainty of market demand stability, the competitor absence, operating in a monopoly regime with no other economic operators.

\section{Aim}

The present research concerns the framework outlined. The paper concerns the application of the PF procedure to a case study related to the expansion of the municipal cemetery of a small town located in Southern Italy. 
The work aims to analyze the procedure for the identification and allocation of the risks associated to the realization and management of PF project among the parties involved - Public Administration and private investor. Furthermore, with reference to the mentioned transformation project, the work intends to verify the feasibility by the Public Administration in the use of the PF operational tool by analyzing the assessment carried out by the proposer subject. The present study has been developed by the authors and commissioned by Public Administration for assessing the effectiveness in the use of PF procedure for the initiative considered.

In this sense, the results of the research could be a support tool for the Public Administration in the decision-making processes aimed to evaluate the PF proposal by the proposer subject in order to ensure an appropriate and detailed investigation on the PPP mechanism. An effective study on the construction, profitability and demand risks of the initiative and a proper description of the economic and financial balance risk represent central phases in the PF convenience assessment.

The paper is structured as follows. In Sect. 3 the different risk typologies connected to a PPP realization and/or renovation project according to Italian legislative references have been illustrated. In Sect. 4 the case study, related to the expansion and the management of the cemetery of a small town located in Southern Italy to be carried out through the PF procedure, has been described. In Sect. 5, the matrix of the main risks associated to the project realization and management considered has been developed to validate the PF initiative. Finally in Sect. 6 the conclusions of the work have been reported.

\section{The Risks Assessment}

In the existing literature concerning the PPP operational tools for urban redevelopment interventions analysis, several contributions are aimed to define the risks for the parties involved in the transformation initiative - public subjects and private investors [4, 9, 12]. In the Italian context of the PPP operational tools, the National Anti-Corruption Authority (ANAC) Guidelines No. 9 indicate the distribution and allocation of initiative risks between the parties involved on the basis of their respective risk management competences [15]. In particular, the ANAC Guidelines No. 9 point out that the contracting authorities have to identify and assess the specific construction and management risks by allocating them to the subjects with the highest control capacities of them. This is confirmed in the reference literature aimed at analyzing the risks components of a PPP projects: for example, Iyer and Sagheer [10] have pointed out that the initiative success depends on the efficient risks transfer to the sector that can best manage them or Grimsey and Lewis [7] have analyzed the risks of PPP arrangements from the perspectives of the various parties, by explaining the contractual relations between the subjects in infrastructure projects and the most performing mechanisms of distributing risks during different phases of the project.

Furthermore, in order to assess the subject ability to manage each risk, the verification of the possibility to adopt suitable measures for the reduction of likely negative effects, i.e. insurance policies, shall be carried out.

The Guidelines include the risks matrix among the PPP contract documents, aimed at regulating ex-ante modalities and limits for the project financial economic conditions review. 
The risks analysis provides to the public administrations a greater awareness of the critical issues that could be arise during the concession period and helps to strengthen the bargaining among the subjects involved - private and public - . In this sense, the risks matrix is part of the made up of contest documents for the tenders evaluation aimed to support the decision processes for the transformation investment implementation. Furthermore, during the execution phase, this document allows the public subject for an adequate monitor on the risks transfer and retention.

In the Italian legislative context, the arts. 3 par. 1 and 180 par. 3 of Legislative Decree No. 50/2016 [13] highlight that in PPP contracts the risk transfer to the private sector implies the allocation to this subject to the construction, availability and demand risks for the entire procedure period. These risk typologies are included in operative risk, i.e. associated to the management of works or services from the demand or the supply side or both points of view.

Moreover, the construction risk, as defined in art. 3 par. 1 of Legislative Decree No. 50/16, is related to delay in delivery times, non-compliance with project standards, increased costs, technical problems and failure to finish the planned work. With regards to this risk category, the ANAC Guidelines No. 9 include the specific risks connected to $i$ ) the design phase, for the occurrence of necessary project variations, resulting from design errors or omissions, such as to significantly affect the time and costs of carrying out the work; ii) an project execution that does not conform to the initial project, linked to failure to comply with the fixed standards; iii) an increase in the cost of production factors or inadequacy or unavailability of those planned in the project; $i v$ ) a wrong costs and construction times assessment; $v$ ) the contractual breaches by suppliers and subcontractors; $v i$ ) the unreliability and inadequacy of the technology used.

The availability risk, as defined in art. 3 par. 1 of Legislative Decree No. 50/16, is associated to the ability, on the part of the concessionaire, to provide the agreed contractual services, both in terms of volume and expected quality standards. The ANAC Guidelines include the risk items related to $i$ ) an extraordinary and not foreseen maintenance, deriving from inadequate design or construction, with a consequent increase in costs; ii) a non-compliance with the performance indicators of the structure or the services provided; $i i i$ ) the total or partial unavailability of the asset and/or of the services to be provided.

In cases of profitable activities, the risk of demand for the services provided for, is associated to the lack of users and, therefore, of cash flows to be obtained in operational phase. The specific risks included in this risks typology concerns $i$ ) the contraction in market demand in terms of a reduction in overall demand for this service; $i i)$ the contraction in specific demand, linked to the occurrence in the reference market of a competitive supply from other operators that negatively affects the current demand.

The attainment of the economic-financial balance (arts. 180, par. 7 and 165 pars. 3, 4, 5 of Legislative Decree 50/2016) represents the fundamental condition for the correct allocation of construction, availability and demand risks in PPP procures. Defined as the simultaneous presence of economic convenience and financial sustainability conditions - art. 3, par. 1 of Legislative Decree 50/2016 -, the economic-financial balance allows to ensure the project capacity to create value during the contract period and to generate an adequate profitability level for the invested capital (economic convenience) 
and to generate sufficient cash flows to guarantee the initial capital repayment (financial sustainability).

The ANAC Guidelines report the main performance indicators for the verification of the economic-financial balance of the initiative - Net Present Value (NPV), Internal Rate of Return (IRR), Revenues and Costs Ratio (R/C), Debt Service Cover Ratio (DSCR) and Long Life Cover Ratio (LLCR).

The initiative bankability strongly affects the risks connected to an economicfinancial imbalance: the resources availability to cover costs on the financial market in fixed time, the sustainability of these resources and the reasonable return on invested capital constitute the funding risk.

With reference to the financial risk, i.e. the risk of an increase in interest rates and/or failure to repay one or more loan installments, with a consequent costs rise or the impossibility of continuing the operation, the analysis of this risk component provides for the assumption of different progressively increasing interest rates to assess effects the main on the economic-financial balance, in terms of performance indicators results.

The whole PPP intervention risks assessment includes the risks connected to the obtaining permits (opinions, licenses, authorizations, etc.) from public and private entities. In this risk category, the commissioning risk is the likelihood that the work will not have approval, from other public subjects or from the community (stakeholders with regard to the intervention to be carried out), with consequent $i$ ) delays in the realization, ii) occurrence of controversies, or in extreme cases, iii) failure of the of the awarding or the entire PPP procedure.

The administrative risk is connected to the considerable delay or refusal in the granting of authorizations by competent public and private entities, or also to the granting of authorizations with additional requirements, by causing delays in the realization phases.

Furthermore, the expropriation risk concerns delays deriving from expropriation operations or higher costs of expropriation due to wrong planning and/or estimation.

In the same risk category, the environmental and/or archaeological risk is linked to the soil characteristics, to the possible reclamation due to soil contamination and to archaeological finds, thereby delays in the project realization and increases in costs for environmental remediation or archaeological protection. Finally, the legislativepolitical-regulatory risk derives from variations in the normative framework and from programmatic policy decisions that cannot be contractually foreseen, by causing increase in costs for adaptation.

The summary of the risks system related to the transformation and management projects carried out through the PF operation tool is rounded off by the default risk - i.e. the likelihood that users are unable to pay the price of the services offered -, the residual value risk - associated to the return of the asset characterized by a lower market value compared to that expected at the end of contractual relationship -, the technical obsoleteness risk - i.e. linked to the faster obsoleteness of plants, by determining higher maintenance costs -, the above and below service interference risk - connected to the different typologies services in the part interested by the intervention (electricity, cables, fiber optics, etc.).

In Table 1 a synthetic overview of the main risk categories illustrated is reported. 
Table 1. The main risk categories in PPP projects

\begin{tabular}{|c|c|}
\hline \multicolumn{2}{|l|}{ Operative risk } \\
\hline \multirow[t]{6}{*}{ Construction risk } & Design risk \\
\hline & Risk of project execution \\
\hline & Risk of increase in production factors cost \\
\hline & Risk of wrong costs and construction times assessment \\
\hline & Risk of contractual breaches by suppliers and subcontractors \\
\hline & Risk of unreliability and inadequacy of the technology used \\
\hline \multirow[t]{3}{*}{ Availability risk } & Risk of extraordinary and not foreseen maintenance \\
\hline & Performance risk \\
\hline & Risks of the asset total or partial unavailability \\
\hline \multirow[t]{2}{*}{ Risk of demand } & Risk of contraction in market demand \\
\hline & Risk of contraction in specific demand \\
\hline \multicolumn{2}{|c|}{ Risk of economic-financial imbalance } \\
\hline \multicolumn{2}{|l|}{ Funding risk } \\
\hline \multicolumn{2}{|l|}{ Financial risk } \\
\hline \multicolumn{2}{|l|}{ Residual value risk } \\
\hline \multicolumn{2}{|c|}{ Commissioning risk } \\
\hline \multicolumn{2}{|l|}{ Administrative risk } \\
\hline \multicolumn{2}{|l|}{ Expropriation risk } \\
\hline \multicolumn{2}{|c|}{ Environmental and/or archaeological risk } \\
\hline \multicolumn{2}{|c|}{ Legislative-political-regulatory risk } \\
\hline \multicolumn{2}{|l|}{ Default risk } \\
\hline \multicolumn{2}{|c|}{ Technical obsoleteness risk } \\
\hline Interference risk & \\
\hline
\end{tabular}

\section{Case Study}

The case study concerns a hypothesis of project financing for the construction of niches and the management for a period of twenty years of the municipal cemetery of a small town located in Southern Italy. The town covers an area of about $40 \mathrm{~km}^{2}$, with a population just over 25,000 inhabitants.

\subsection{Description of the Cemetery Expansion and Management Project}

The project proposed provides for the construction of cemetery niches in the area established for the expansion of the municipal cemetery and their subsequent management. The initiative plays a strategic role for the small town due to the current needs, equal to 
476 niches, and to the centrality of this service for the community, as confirmed by the inclusion of the intervention in the Municipal 2017-2019 public works Programming Document.

In particular, the project concerns the realization of 3,968 niches divided into five lots - lot A composed by 812 niches to be built in the first year of the management period, lot B of 2,176 in the second year of the management period, lot $\mathrm{C}$ of 140 niches in the fourteenth year of the management period, lot D1 constituted by 448 niches to be realized in the fifteenth year of the entire period considered equal to twenty years and lot D2 of 392 niches in the seventeenth year.

The niches will be built with reinforced concrete walls on a continuous foundation consisting of a reinforced concrete slab. The external shell and the tombstones will be built by using of local materials and the roof will be protected by two layers of waterproofing membrane. The project also provides for the modernization of the pedestrian viability through a paving in pressed bricks, such as to those already existing in other cemetery areas. The trees in the area intended to the niches in lot D will be explanted and replanted in a suitable cemetery spaces.

The project envisages different measures aimed at optimizing management costs, such as the inclusion of votive LED lights, and at allowing the accessibility to the people with low modality, thanks to the absence of architectural barriers and to the presence of an electric vehicle to facilitate the users' movement.

\subsection{The PF Procedure}

The hypothesis assumed is that the Public Administration and a private investor conclude an onerous and written agreement for a fixed time period - equal to twenty years -, in order to expand and manage the municipal cemetery. The PF procedure provides that the new construction interventions are carried out by the private investor, in exchange for the temporary use of the new portion of cemetery for the fixed time period. In particular, under this assumption the private investor bears the realization costs and manages the new cemetery portion for the entire twenty-year concession period. The revenue items for the private investor will derive from the niches concession, whereas for the Public Administration a royalty equal to $250 €$ for each niche has been defined to be paid.

The verification of the effective use of PF in terms of financial feasibility from the private investor point of view related to intervention is carried out through the cash flows analysis.

With reference to the case study analyzed, the cash flow analysis has been developed by proposer subject and it shall be validated by the authors to verify the outputs obtained in terms of intervention profitability and risks. Starting from the market demand description, by considering the resident population trend and the National Institute of Statistics (ISTAT) mortality and birth rates [18] (Fig. 1), in the cash flows analysis, for each concession year, the costs (investment and management) and the revenues have been assessed and the performance indicators have been determined.

Finally, for the assessment of the risk linked to the economic-financial balance, a cash flows analysis, named new analysis, has been developed. It should be observed that some hypothesis have been borrowed from the proposer subject analysis, as considered valid: if a different hypothesis has been introduced in the Manuscript it is highlighted. 


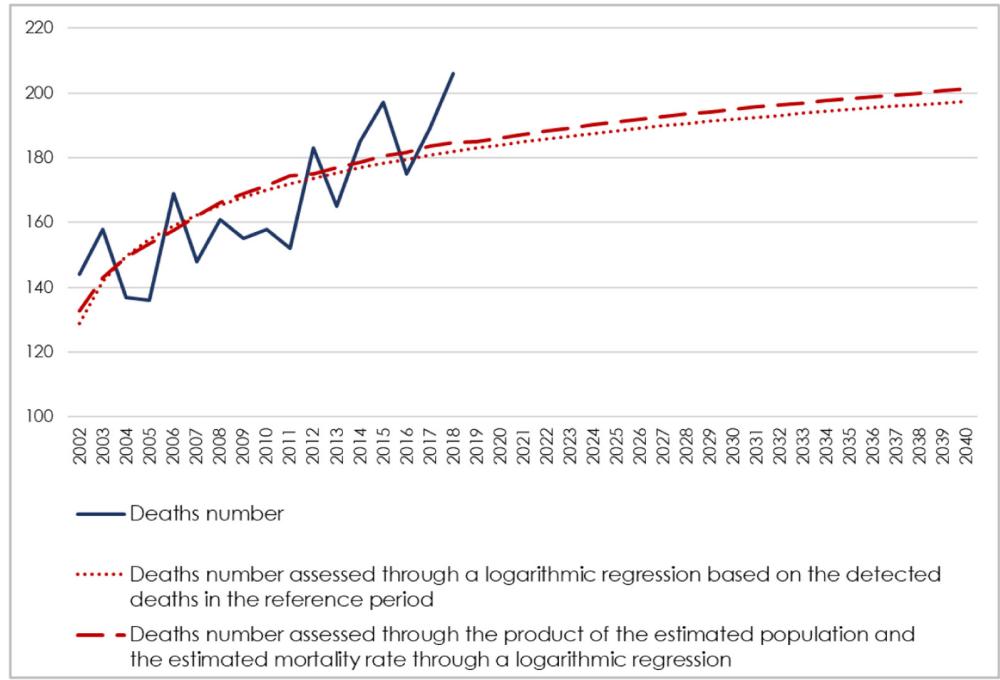

Fig. 1. Number of deaths detected and estimated

Thus, with reference to the case study, the main assumptions of the new analysis can be summarized as follows:

- the period of the analysis is equal to twenty years;

- the construction costs are assessed by considering the unitary costs reported in Regional Price List of Public Works of the year 2019. In the absence of the unitary price, the new realization costs are assessed by price lists of public and private works, currently used in the Region and by the data reported in the "Building typology prices" list [6], and are validated by consulting local operators and construction companies. Moreover, the unitary construction cost related to the case study cemetery project niches is consistent with the parametric unitary costs related to other similar Italian cemetery projects, recently built. In the proposer subject cash flows analysis the total construction cost was assessed equal to 3,500,000 €, whereas in the new cash flows analysis the total construction cost assessed is $3,700,000 €$, by determining a percentage variation equal to $\pm 6 \%$. In particular, in the new analysis the estimated amounts for the different project components are higher compared those calculated by the proposer subject, with increases that vary from $+4 \%$ to $+14 \%$, by depending on the lots (Fig. 2). This variation is in line with the difference in spending capacity between an ordinary investor, i.e. the cost assessment related to the reference price lists, and the specific investor, i.e. the proposer subject.

- the $36 \%$ of the investment costs related to the niches realizations intervention are borrowed from a credit institution, through a 20-year mortgage to be returned through constant annual down payments with an interest rate equal to $5.00 \%$; 


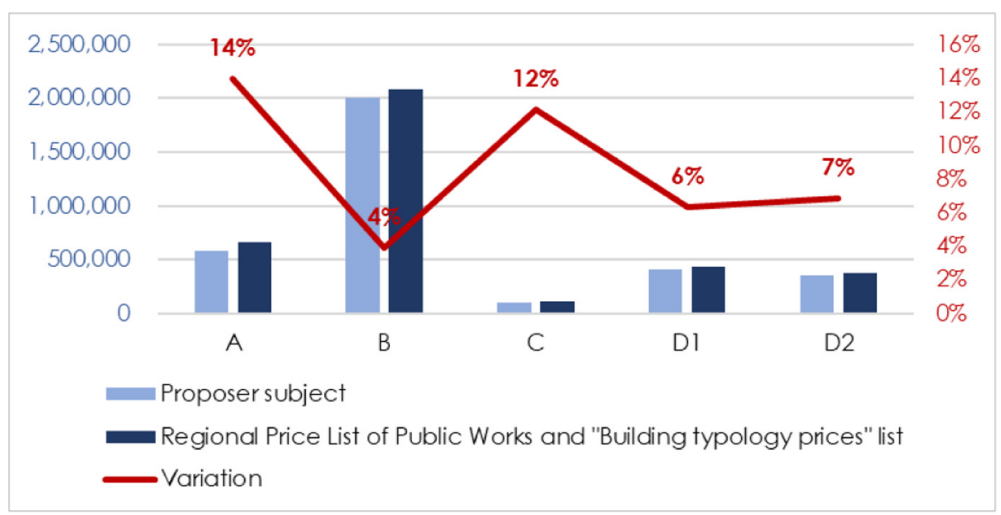

Fig. 2. Comparison between costs estimated by the proposer subject and costs assessed through the price lists

- the technical costs - for the definitive plan, a draft convention, the economic-financial plan, the specification of the service characteristic and of the management - are assessed equal to $60,000 €$;

- the general expanses - including the fees for specialized technicians and professionals, the costs of the insurance for the construction phase, etc. - are assessed equal to $100,000 €$;

- the management costs include the expenses for equipment, the ordinary maintenance costs, the personnel and insurance costs, the expenses for utilities. These costs are assessed by considering those currently borne by Public Administration for the existing cemetery portion;

- the revenue items concern the niches concession and the services as burial, boneswashing, votive lights, etc. The revenues are determined following the demand curve assessed. The market demand depends on two main factors: $i$ ) the number of deaths expected during the concession period, by deducting the burials, cremations or dead to be placed in private chapels $i$ ) the previous market demand ( 476 niches). Therefore, on the basis of the estimations carried out, the annual deaths number has been estimated equal to 162 , i.e. the annual expected average deaths number - equal to 190 - reduced by $15 \%$ by considering the potential cremations, burials and dead to be placed in private chapels. The amount estimated is different from the proposer subject analysis (180 niches/year) and represents a prudential initial data for the cash flow assessment. With regards to the tariffs implemented by the proposer subject in his analysis for the niches concession and for installation of slabs and accessories are equal to 2,500 $-2,600 € /$ niche. This amount is consistent with the current ordinarily tariffs charged in the specific reference context by Public Administration and, therefore, they are retained in the new analysis;

- for each niche, a royalty equal to $250 €$ has been defined to be paid to the Public Administration: this monetary amount is include in the tariff paid for the niches concession; 
- the discounting rate is fixed equal to 7\% (in proposer subject analysis it was set equal to 5\%). This amount has been determined taking into account the risks of similar initiatives in the reference market.

Finally, the comparison between the performance indicators - NPV, IRR and R/Cassessed in the new analysis and those determined by proposer subject has been performed, in order to verify the financial feasibility of the initiative in stressed situations (with more prudential assumptions).

In Table 2, the NPV, IRR and R/C obtained in the new analysis and in the proposer subject analysis are reported.

Table 2. Performance indicators determined in proposer subject analysis and in the new analysis.

\begin{tabular}{l|l|l}
\hline Indicator & Proposer subject analysis & New analysis \\
\hline NPV $(€)$ & $2,100,000$ & $1,200,000$ \\
\hline IRR & $36.2 \%$ & $32.4 \%$ \\
\hline R/C & 1.5 & 1.2 \\
\hline
\end{tabular}

It should be observed that the economic-financial balance is ensure in the stressed situation assumed in the new analysis. However, the likelihood that the economic-financial balance is not ensured is high, by considering a possible variation of the taxes and duties system, of regulatory framework, the longer time for the granting of authorizations and unforeseen geological or geotechnical characteristics.

\section{The Risks Matrix}

In order to assess the different risk categories related to the expansion and management project of the cemetery considered in the present analysis, the matrix risks has been developed.

The Table 3 reports a framework of the main risk typologies connected to the project. The investigation allows to carry out an exhaustive analysis of the risks transfer to the various subjects involved in the initiative - private and public sectors -, by explaining the likely root causes, the specific strategic measures aimed at avoiding or, at least, at reducing the effects of negative phenomenon. With reference to each risk item illustrated in Sect. 3 of the present paper, in the Table 2 a qualitative indication of the likelihood that it occurs, the economic effects deriving from its occurrence and the subject to whom the risk is transferred are shown. In particular, the likelihood of each risk occurring has been evaluated by using a verbal scale, as such indicated by ANAC Guidelines No. 9 - nil, minimum, low, medium and high. The assessment process by which the likelihood of risk has been measured, is included in the Table through a synthetic description of the specific aspect of the intervention: thus, the each intervention critical issue and weakness is explored in order to identify and manage the most complex and risky elements of the PF initiative considered. 
Table 3. Risks matrix related to the cemetery expansion and management project.

\begin{tabular}{|c|c|c|c|}
\hline Risk typology & Likelihood of risk occurring & $\begin{array}{l}\text { Economic } \\
\text { effects assessed }\end{array}$ & $\begin{array}{l}\text { Subject to } \\
\text { whom the } \\
\text { risk is } \\
\text { transferred }\end{array}$ \\
\hline $\begin{array}{l}\text { Project design and execution } \\
\text { risks }\end{array}$ & $\begin{array}{l}\text { Low } \\
\text { The standardized building typology, } \\
\text { associated to specific construction } \\
\text { indications, makes it unlikely } \\
\text { dissimilarities between the project } \\
\text { planned and the work completed }\end{array}$ & $\begin{array}{l}+8 \% \text { of the } \\
\text { construction } \\
\text { costs }\end{array}$ & Private \\
\hline $\begin{array}{l}\text { Risk of increase in production } \\
\text { factors cost and risk of wrong } \\
\text { costs and construction times } \\
\text { assessment }\end{array}$ & $\begin{array}{l}\text { Medium-low } \\
\text { The technical specifications of the } \\
\text { project detect a low level of } \\
\text { technical-realization complexity. } \\
\text { However, in a concession period of } \\
\text { twenty years a costs increase is } \\
\text { plausible }\end{array}$ & $\begin{array}{l}+6 \% \text { of the } \\
\text { assessed costs }\end{array}$ & Private \\
\hline $\begin{array}{l}\text { Risk of the contractual } \\
\text { breaches by suppliers and } \\
\text { sub-contractors }\end{array}$ & Not estimated & & Private \\
\hline $\begin{array}{l}\text { Risks of unreliability and } \\
\text { inadequacy of the technology } \\
\text { used }\end{array}$ & $\begin{array}{l}\text { Nil } \\
\text { The construction technologies are } \\
\text { easy to implement and the plant } \\
\text { component is limited, consolidated } \\
\text { and widespread }\end{array}$ & & \\
\hline $\begin{array}{l}\text { Risk of an extraordinary and } \\
\text { not foreseen maintenance }\end{array}$ & $\begin{array}{l}\text { High } \\
\text { In the PF proposal carried out by } \\
\text { proposer subject there is no explicit } \\
\text { reference to extraordinary } \\
\text { maintenance. However, in the } \\
\text { ordinary maintenance, to be borne by } \\
\text { the private investor, only the } \\
\text { interventions aimed at contrasting the } \\
\text { ordinary usury of structures and } \\
\text { plant, the equipment and cleaning } \\
\text { materials costs are included }\end{array}$ & $\begin{array}{l}+1 \% \text { of the } \\
\text { assessed costs }\end{array}$ & Public \\
\hline $\begin{array}{l}\text { Performance and asset total or } \\
\text { partial unavailability risks }\end{array}$ & $\begin{array}{l}\text { Low } \\
\text { The construction complexity is } \\
\text { limited and the proposer subject is } \\
\text { suitable for the project realization } \\
\text { both in terms of quantity and } \\
\text { expected quality standards. The } \\
\text { management model is consistent with } \\
\text { similar activities carried out in } \\
\text { nearby towns by the proposer subject }\end{array}$ & & Private \\
\hline
\end{tabular}


Table 3. (continued)

\begin{tabular}{|c|c|c|c|}
\hline Risk typology & Likelihood of risk occurring & $\begin{array}{l}\text { Economic } \\
\text { effects assessed }\end{array}$ & $\begin{array}{l}\text { Subject to } \\
\text { whom the } \\
\text { risk is } \\
\text { transferred }\end{array}$ \\
\hline $\begin{array}{l}\text { Risk of contraction in market } \\
\text { demand }\end{array}$ & $\begin{array}{l}\text { Medium-low } \\
\text { As a precaution, in the assessment } \\
\text { the widespread phenomenon of } \\
\text { requesting a concession for niches } \\
\text { before actual use has not been } \\
\text { considered }\end{array}$ & $\begin{array}{l}\text { In the proposer } \\
\text { subject } \text { analysis } \\
\text { ( } 180 \\
\text { niches/year) at } \\
\text { the last } \\
\text { concession } \\
\text { period } 414 \\
\text { unallocated } \\
\text { niches will be, } \\
\text { i.e. a foregone } \\
\text { revenues of } \\
\text { about } 870,000 \\
€ \text { for the } \\
\text { private investor } \\
\text { and a loss of } \\
\text { royalty of about } \\
100,000 € \text { for } \\
\text { the Public } \\
\text { Administration }\end{array}$ & $\begin{array}{l}\text { Private } \\
\text { and public }\end{array}$ \\
\hline $\begin{array}{l}\text { Risk of contraction in specific } \\
\text { demand }\end{array}$ & $\begin{array}{l}\text { Nil } \\
\text { There are no possible competitors: } \\
\text { the regime provided is unilateral } \\
\text { monopoly characterized by a single } \\
\text { supplier (i.e. the Public } \\
\text { Administration through the private } \\
\text { investor) and numerous applicants } \\
\text { (i.e. the relatives of the deceaseds) }\end{array}$ & & \\
\hline $\begin{array}{l}\text { Risk of economic-financial } \\
\text { imbalance }\end{array}$ & $\begin{array}{l}\text { High } \\
\text { The likelihood that the } \\
\text { economic-financial balance is not } \\
\text { determined is high, by considering a } \\
\text { possible variation of the taxes and } \\
\text { duties system, of regulatory } \\
\text { framework, the longer time for the } \\
\text { granting of authorisations and } \\
\text { unforeseen geological or } \\
\text { geotechnical characteristics }\end{array}$ & $\begin{array}{l}\text { NPV: }-1 \\
\text { million } € \text { TIR: } \\
-4 \%\end{array}$ & Public \\
\hline Funding risk & $\begin{array}{l}\text { Medium } \\
\text { The credit institution shall declare its } \\
\text { interest and availability to finance the } \\
\text { initiative, by specifying the monetary } \\
\text { amount }\end{array}$ & +1.4 million $€$ & Private \\
\hline Financial risk & $\begin{array}{l}\text { Low } \\
\text { The possibility related to an increase } \\
\text { of interest taxes is remote, as it is } \\
\text { connected to macroeconomic } \\
\text { conditions variations }\end{array}$ & $\begin{array}{l}+100,000 € \text { of } \\
\text { interests for } \\
\text { increasing of } \\
\text { interest rate of } \\
50 \mathrm{bps}\end{array}$ & Private \\
\hline
\end{tabular}


Table 3. (continued)

\begin{tabular}{|c|c|c|c|}
\hline Risk typology & Likelihood of risk occurring & $\begin{array}{l}\text { Economic } \\
\text { effects assessed }\end{array}$ & $\begin{array}{l}\text { Subject to } \\
\text { whom the } \\
\text { risk is } \\
\text { transferred }\end{array}$ \\
\hline Residual value risk & $\begin{array}{l}\text { Medium-low } \\
\text { Assuming } i) 162 \text { niches/year granted, } \\
\text { ii) } 500 € / \text { niches for the tariff for the } \\
\text { renewal of the concession contract } \\
\text { after the period of } 99 \text { years, iii) } 7 \% \\
\text { for the discounting rate fixed, at the } \\
\text { end of the concession the residual } \\
\text { value is equal about to } 4,795 € \text {, to } \\
\text { which correspond a present value of } \\
1,240 \text { ( } 1 \text { January } 2021) \text {. At the end of } \\
\text { concession period, the management } \\
\text { and maintenence costs will not bear } \\
\text { to Public Administration but they } \\
\text { will bear to another private subject. } \\
\text { In the residual value assessment, } \\
\text { possible niches not granted for which } \\
\text { the full concession tariffs, in addition } \\
\text { to the royalty equal to } 250 € \text { for the } \\
\text { Public Administration, have not been } \\
\text { considered }\end{array}$ & $\begin{array}{l}\text { At the end of } \\
\text { the concession, } \\
\text { the proposer } \\
\text { subject } \text { assumes } \\
\text { that all niches } \\
\text { will be granted. } \\
\text { The residual } \\
\text { value is } \\
\text { constituted } \\
\text { exclusively by } \\
\text { the discounted } \\
\text { value deriving } \\
\text { from the } \\
\text { renewal of the } \\
\text { concession for a } \\
\text { further } 99 \text { years, } \\
\text { in compliance } \\
\text { with the } \\
\text { provisions of } \\
\text { Presidential } \\
\text { Decree } \\
285 / 1990\end{array}$ & \\
\hline Commissioning risk & $\begin{array}{l}\text { Low } \\
\text { There are no public entities that can } \\
\text { hinder the project implementation } \\
\text { and the community has informally } \\
\text { expressed a positive opinion on the } \\
\text { initiative, given the existing } \\
\text { unsatisfied demand }\end{array}$ & & Public \\
\hline Administrative risk & $\begin{array}{l}\text { Low } \\
\text { The intended use of the property laid } \\
\text { down in land-use plan is consistent } \\
\text { with the function planned in the } \\
\text { intervention }\end{array}$ & & Public \\
\hline Expropriation risk & $\begin{array}{l}\text { Almost nil } \\
\text { It is not necessary to proceed to } \\
\text { expropriation since the area is already } \\
\text { intended to municipal cemetery }\end{array}$ & & Public \\
\hline
\end{tabular}


Table 3. (continued)

\begin{tabular}{l|l|l|l}
\hline Risk typology & Likelihood of risk occurring & $\begin{array}{l}\text { Economic } \\
\text { effects assessed }\end{array}$ & $\begin{array}{l}\text { Subject to } \\
\text { whom the } \\
\text { risk is } \\
\text { transferred }\end{array}$ \\
\hline $\begin{array}{l}\text { Environmental and/or } \\
\text { archaeological risk }\end{array}$ & $\begin{array}{l}\text { Low } \\
\text { There are no environmental } \\
\text { constraints and, given the intended } \\
\text { use and location of the intervention } \\
\text { area, there is no incompatibility } \\
\text { between the project and the land use }\end{array}$ & Public \\
\hline Legislative-political-regulatory & $\begin{array}{l}\text { Almost nil } \\
\text { risk unsatisfied need to expand the } \\
\text { municipal cemetery and the absence } \\
\text { of costs to be borne by public subject } \\
\text { make nil this risk typology }\end{array}$ & $\begin{array}{l}\text { Medium-high } \\
\text { Although the proposed tariffs are } \\
\text { consistent with those current applied } \\
\text { by Public Administration for the } \\
\text { niches concession, the tariffs review } \\
\text { mechanism defined by proposer } \\
\text { subject afford to uncontrollably } \\
\text { increase them }\end{array}$ & Public \\
\hline Default risk & $\begin{array}{l}\text { Low } \\
\text { The planned plants will built with } \\
\text { consolidated construction } \\
\text { technologies: there is no risk of } \\
\text { technical obsolescence, especially by } \\
\text { considering effective maintenance } \\
\text { interventions measures }\end{array}$ & $\begin{array}{l}\text { Almost nil } \\
\text { In the area intervention, the presence } \\
\text { of various services (electricity, } \\
\text { cables, fiber optics, etc.) is confirmed } \\
\text { by official documents }\end{array}$ & Public \\
\hline Interference risk & \begin{tabular}{l} 
Public \\
\hline Technical obsoleteness risk
\end{tabular} & & \\
\hline
\end{tabular}

\section{Conclusions}

In the PPP context, the PF operational tool represents an effective procedure to carry out cemetery expansion, new realization and/or redevelopment interventions in Italian cities. The risks matrix constitutes a fundamental document among those place made up of contest, as it is relevant for the proposal/proposals assessment. The identification of the risks associated to the project and the explanation of the each risk occurring likelihood allows to analyze in a detailed manner the initiative and to allocate the different risks typology between the subjects involved - Public Administration and private investor. Furthermore, the risks matrix helps a constant monitoring of risks retention during the construction and management phases in order to avoid or, at least, to reduce the effects 
of negative occurrences that could led to the initiative failure, i.e. increase in costs or construction time or, in extreme case, interruption of the works.

The case study considered in the present research has concerned a hypothesis of project financing for the construction of niches and the management for a period of twenty years of the municipal cemetery of a small town located in Southern Italy. For each risk category, as regulated by Italian legislative references (Legislative Decree No. 50/2016 and ANAC Guidelines No. 9) the likelihood of its occurring has been assessed by using a verbal scale, in order $i$ ) to provide for an exhaustive framework of the different project components, $i i)$ to give specific indications to the Public Administration and the private investor about the respective risk items that should check and iii) to support the risk management phase. The research has pointed out the relevance of risks assessment from the preliminary design stages able to identify likely critical issues of the project and to define the PPP agreement terms for parties involved. In particular, it should be highlighted that the low risk of the intervention - due to the absence of demand risk, to the constructive simplicity, etc. - allows the PF to be a useful tool for the construction and management of cemetery projects, as confirmed by the numerous situations in which it has been applied. Further insights of the research may concern the development of a more detailed Cost-Benefit analysis for the social impacts investigation of the initiative. As the case study shows, the main limitations of the risk matrix concern: $i$ ) the use of verbal sentences which could be inadequately interpreted; $i i$ ) the absence of a summary indicator of the various risk typologies. To overcome these limits an improvement of risks matrix could be carried out, for example by assessing the likelihood of risks occurring through quantitative measures and indicators and by aggregating the results through a multi-criteria approach, in order to compare different project proposals too.

\section{References}

1. Aziz, A.M.A.: Successful delivery of public private partnerships for infrastructure development. J. Constr. Eng. Manag. 133(12), 918-931 (2007)

2. Calabrò, F., Della Spina, L.: La fattibilità economica dei progetti nella pianificazione strategica, nella progettazione integrata, nel cultural planning, nei piani di gestione. Un modello sperimentale per la valorizzazione di immobili pubblici in Partenariato Pubblico Privato. LaborEst 16 (2018)

3. Calabrò, F., Della Spina, L.: The public-private partnership for the enhancement of unused public buildings: an experimental model of economic feasibility project. Sustainability 11(20), 5662 (2019)

4. Chen, H., Zhang, L., Wu, X.: Performance risk assessment in public-private partnership projects based on adaptive fuzzy cognitive map. Appl. Soft Comput. 93, 106413 (2020)

5. Edili, A.N.C.: Il Project Financing in Italia - L'indagine ANCE sulla realizzazione delle opere (2012). Accessed 23 June 2014

6. Engineers and Architects Register of Milan: Prezzi Tipologie Edilizie. DEI Tipografia del Genio Civile. Quine Business Publisher (2019)

7. Grimsey, D., Lewis, M.K.: Evaluating the risks of public private partnerships for infrastructure projects. Int. J. Proj. Manag. 20(2), 107-118 (2002)

8. Heravi, G., Hajihosseini, Z.: Risk allocation in public-private partnership infrastructure projects in developing countries: case study of the Tehran-Chalus Toll Road. J. Infrastruct. Syst. 18(3), 210-217 (2012) 
9. Iqbal, S., Choudhry, R.M., Holschemacher, K., Ali, A., Tamošaitienė, J.: Risk management in construction projects. Technol. Econ. Dev. Econ. 21(1), 65-78 (2015)

10. Iyer, K.C., Sagheer, M.: Hierarchical structuring of PPP risks using interpretative structural modeling. J. Constr. Eng. Manag. 136(2), 151-159 (2010)

11. Jefferies, M., McGeorge, W.D.: Using public-private partnerships (PPPs) to procure social infrastructure in Australia. Eng. Constr. Archit. Manag. 16(5), 415-437 (2009)

12. Keçi, J.: Public private partnership for infrastructure projects: mapping the key risks. Int. J. Soc. Behav. Educ. Econ. Bus. Ind. Eng. 9(9), 3141-3151 (2015)

13. Legislative Decree No. $50 / 2016$

14. Morano, P., Tajani, F., Guarini, M. R., Di Liddo, F.: An evaluation model for the definition of priority lists in PPP redevelopment initiatives. In: Bevilacqua, C., Calabrò, F., Spina, L.D. (eds.) New Metropolitan Perspectives, vol. 178, pp. 451-461. Springer, Cham (2020). https:// doi.org/10.1007/978-3-030-48279-4_43

15. National Anti-Corruption Authority (ANAC): Guidelines No. 9 - Monitoring of contracting administrations on the economic operator activities in public-private partnership contracts (2018)

16. Scano, D.: Project financing: società e impresa, p. 288. Giuffrè Editore (2006)

17. Shen, L.Y., Platten, A., Deng, X.P.: Role of public private partnerships to manage risks in public sector projects in Hong Kong. Int. J. Proj. Manag. 24(7), 587-594 (2006)

18. www.istat.it 